\title{
Stability Theory for Hybrid Dynamical Systems
}

\author{
Hui Ye, Anthony N. Michel, Fellow, IEEE, and Ling Hou
}

\begin{abstract}
Hybrid systems which are capable of exhibiting simultaneously several kinds of dynamic behavior in different parts of a system (e.g., continuous-time dynamics, discrete-time dynamics, jump phenomena, switching and logic commands, and the like) are of great current interest. In the present paper we first formulate a model for hybrid dynamical systems which covers a very large class of systems and which is suitable for the qualitative analysis of such systems. Next, we introduce the notion of an invariant set (e.g., equilibrium) for hybrid dynamical systems and we define several types of (Lyapunov-like) stability concepts for an invariant set. We then establish sufficient conditions for uniform stability, uniform asymptotic stability, exponential stability, and instability of an invariant set of hybrid dynamical systems. Under some mild additional assumptions, we also establish necessary conditions for some of the above stability types (converse theorems). In addition to the above, we also establish sufficient conditions for the uniform boundedness of the motions of hybrid dynamical systems (Lagrange stability). To demonstrate the applicability of the developed theory, we present specific examples of hybrid dynamical systems and we conduct a stability analysis of some of these examples (a class of sampleddata feedback control systems with a nonlinear (continuous-time) plant and a linear (discrete-time) controller, and a class of systems with impulse effects).
\end{abstract}

Index Terms - Asymptotic stability, boundedness, dynamical system, equilibrium, exponential stability, hybrid, hybrid dynamical system, hybrid system, instability, invariant set, Lagrange stability, Lyapunov stability, stability, ultimate boundedness.

\section{INTRODUCTION}

$\mathbf{H}$ YBRID SYSTEMS which are capable of exhibiting simultaneously several kinds of dynamic behavior in different parts of the system (e.g., continuous-time dynamics, discrete-time dynamics, jump phenomena, logic commands, and the like) are of great current interest (see, e.g., [1]-[9]). Typical examples of such systems of varying degrees of complexity include computer disk drives [4], transmission and stepper motors [3], constrained robotic systems [2], intelligent vehicle/highway systems [8], sampled-data systems [10]-[12], discrete event systems [13], switched systems [14], [15], and many other types of systems (refer, e.g., to the papers included in [5]). Although some efforts have been made to provide a unified framework for describing such systems (see, e.g., [9] and [29]), most of the investigations in the literature focus on specific classes of hybrid systems. More to the point, at

Manuscript received August 2, 1996. Recommended by Associate Editor, P. J. Antsaklis. This work was supported in part by the National Science Foundation under Grant ECS93-19352.

$\mathrm{H}$. Ye is with the Wireless Technology Laboratory at Lucent Technologies, Whippany, NJ 07109 USA.

A. N. Michel and L. Hou are with the Department of Electrical Engineering, University of Notre Dame, Notre Dame, IN 46556 USA (e-mail: Anthony.N.Michel.1@nd.edu).

Publisher Item Identifier S 0018-9286(98)02806-2. the present time, there does not appear to exist a satisfactory general model for hybrid dynamical systems which is suitable for the qualitative analysis of such systems. As a consequence, a general qualitative theory of hybrid dynamical systems has not been developed thus far. In the present paper we first formulate a model for hybrid dynamical systems which covers a very large class of systems. In our treatment, hybrid systems are defined on an abstract time space which turns out to be a special completely ordered metric space. When this abstract time space is specialized to the real time space (e.g., $R^{+}=[0, \infty)$, or $\left.N=\{0,1, \cdots\},\right)$, then our definition of a hybrid dynamical system reduces to the usual definition of general dynamical system (see, e.g., [16, p. 31]).

For dynamical systems defined on abstract time space (i.e., for hybrid dynamical systems) we define various qualitative properties (such as Lyapunov stability, asymptotic stability, and so forth) in a natural way. Next, we embed the dynamical system defined on abstract time space into a general dynamical system defined on $R^{+}$, with qualitative properties preserved, using an embedding mapping from the abstract time space to $R^{+}$. The resulting dynamical system (defined now on $R^{+}$) consists in general of discontinuous motions.

The Lyapunov stability results for dynamical systems defined on $R^{+}$in the existing literature require generally continuity of the motions (see, e.g., [16]-[19]), and as such, these results cannot be applied directly to the discontinuous dynamical systems described above. We establish in the present paper results for uniform stability, uniform asymptotic stability, exponential stability, and instability of an invariant set (such as, e.g., an equilibrium) for such discontinuous dynamical systems defined on $R^{+}$and hence for the class of hybrid dynamical systems considered herein. These results provide not only sufficient conditions, but also some necessary conditions, since converse theorems for some of these results are established under some very minor additional assumptions. In addition to the above, we also establish sufficient conditions for the uniform boundedness and uniform ultimate boundedness of the motions of hybrid dynamical systems (Lagrange stability).

Existing results on hybrid dynamical systems seem to have been confined mostly to finite-dimensional models. We emphasize that the present results are also applicable in the analysis of infinite-dimensional systems.

We apply the preceding results in the stability analysis of a class of sampled-data feedback control systems consisting of an interconnection of a nonlinear plant (described by a system of first order ordinary differential equations) and a linear digital controller (described by a system of first-order linear difference equations). The interface between the plant and the controller is an A/D converter, and the interface 
between the controller and the plant is a D/A converter. The qualitative behavior of sampled-data feedback control systems has been under continual investigation for many years, with an emphasis on linear systems (see [10]-[12]). For the present example we show that under reasonable conditions the qualitative behavior of the nonlinear sampled-data feedback system can be deduced from the qualitative behavior of the corresponding linearized sampled-data feedback system. Although this result has been obtained by methods other than the present approach [26], [32], we emphasize that our objective here is to demonstrate an application of our theory to a well-known class of problems.

In addition to sampled-data feedback control systems, we apply the results developed herein in the stability analysis of a class of systems with impulse effects. For this class of systems, the results presented constitute improvements over existing results. We have also analyzed a class of switched systems by the present results. However, due to space limitations, these were not included.

For precursors of our results reported herein, as well as additional related materials not included here (due to space limitations), please refer to [22]-[28] and [30].

\section{NOTATION}

Let $R$ denote the set of real numbers and let $R^{n}$ denote real $n$-space. If $x \in R^{n}$, then $x^{T}=\left(x_{1}, \cdots, x_{n}\right)$ denotes the transpose of $x$. Let $R^{n \times m}$ denote the set of real $n \times m$ matrices. If $B=\left[b_{i j}\right]_{n \times m} \in R^{n \times m}$, then $B^{T}$ denotes the transpose of B. A matrix $A \in R^{n \times n}$ is said to be Schurstable if all eigenvalues of $A$ are within the unit circle of the complex plane. For $x \in R^{n}$, let $\|x\|$ denote the Euclidean vector norm, $\|x\|=\left(x^{T} x\right)^{1 / 2}$, and for $A \in R^{n \times n}$, let $\|A\|$ denote the norm of $A$ induced by the Euclidean vector norm, i.e., $\|A\|=\left[\lambda_{\max }\left(A^{T} A\right)\right]^{\frac{1}{2}}$.

Let $R^{+}$denote the set of nonnegative real numbers, i.e., $R^{+}=[0,+\infty)$, and let $N$ denote the set of nonnegative integers, i.e., $N=\{0,1, \cdots$,$\} . For any r \in R^{+},[r]$ denotes the greatest integer which is less than or equal to $r$. Let $X$ be a subset of $R^{n}$ and let $Y$ be a subset of $R^{m}$. We denote by $C[X, Y]$ the set of all continuous functions from $X$ to $Y$, and we denote by $C^{k}[X, Y]$ the set of all functions from $X$ to $Y$ which have continuous derivatives up to and including order $k$.

A set $T$ is said to be completely ordered with the order relation " $\prec$ " if for any $t_{1}, t_{2} \in T$ and $t_{1} \neq t_{2}$, either $t_{1} \prec t_{2}$ or $t_{2} \prec t_{1}$. We let $(T, \rho)$ be a metric space where $T$ represents the set of elements of the metric space and $\rho$ denotes the metric.

We denote a mapping $f$ of a set $V$ into a set $W$ by $f: V \rightarrow W$, and we denote the set of all mappings from $V$ into $W$ by $\{V \rightarrow W\}$.

We say that a function $\phi \in C\left[[0, r], R^{+}\right]$(respectively, $\phi \in$ $C\left[R^{+}, R^{+}\right]$) belongs to class $K$ (i.e., $\phi \in K$ ), if $\phi(0)=0$ and if $\phi$ is strictly increasing on $[0, r]$ [respectively, $[0, \infty)]$. We say that $\phi \in C\left[R^{+}, R^{+}\right]$belongs to class $K R$ if $\phi \in K$ and if $\lim _{s \rightarrow \infty} \phi(s)=\infty$. A continuous function $\sigma:\left[s_{1}, \infty\right) \rightarrow R^{+}$ is said to belong to Class $L$ if $\sigma$ is strictly decreasing on $\left[s_{1}, \infty\right)$ and if $\lim _{s \rightarrow \infty} \sigma(s)=0$ where $s_{1} \in R^{+}$.

\section{HYBRID DYNAMICAL SYSTEMS}

The present section consists of three parts.

\section{A. Hybrid Systems}

We require the following notion of time space.

Definition 3.1 (Time Space): A metric space $(T, \rho)$ is called a time space if:

1) $T$ is completely ordered with order “ ‘ $\prec$;",

2) $T$ has a minimal element $t_{\min } \in T$, i.e., for any $t \in T$ and $t \neq t_{\min }$ it is true that $t_{\min } \prec t$;

3) for any $t_{1}, t_{2}, t_{3} \in T$ such that $t_{1} \prec t_{2} \prec t_{3}$, it is true that $\rho\left(t_{1}, t_{3}\right)=\rho\left(t_{1}, t_{2}\right)+\rho\left(t_{2}, t_{3}\right)$;

4) $T$ is unbounded from above, i.e., for any $M>0$, there exists a $t \in T$ such that $\rho\left(t, t_{\min }\right)>M$.

When $\rho$ is clear from context, we will frequently write $T$ in place of $(T, \rho)$.

Definition 3.2 (Equivalent Time Spaces): For two time spaces $T, \hat{T}$, we say that $T$ and $\hat{T}$ are equivalent (with respect to $h$ ) if there exists a mapping $h: T \rightarrow \hat{T}$ such that $h$ is an isometric mapping from $T$ to $\hat{T}$, and such that the order relations in $T$ and $\hat{T}$ are preserved under $h$. Henceforth, we use the notation $T \sim \hat{T}$ to indicate that $T$ and $\hat{T}$ are equivalent. In addition, for $T_{0} \subset T, \hat{T}_{0} \subset \hat{T}$, we use the notation $\left(T, T_{0}\right) \sim\left(\hat{T}, \hat{T}_{0}\right)$ to indicate that $T$ and $\hat{T}$ are equivalent (with respect to $h$ ), and $T_{0}$ and $\hat{T}_{0}$ are equivalent (with respect to $h_{T_{0}}$ ), where $h_{T_{0}}$ is the restriction of $h$ to $T_{0}$.

We can now introduce the concept of motion defined on a time space $(T, \rho)$.

Definition 3.3 (Motion): Let $(X, d)$ be a metric space and let $A \subset X$. Let $(T, \rho)$ be a time space, and let $T_{0} \subset T$. For any fixed $a \in A, t_{0} \in T_{0}$, we call a mapping $\tilde{p}\left(\cdot, a, t_{0}\right)$ : $\tilde{T}_{a, t_{0}} \rightarrow X$ a motion on $T$ if:

1) $\tilde{T}_{a, t_{0}}$ is the subset of a time space $\tilde{T}$ (in general not equal to $T$ ) which is determined by $\left(a, t_{0}\right)$, and $\left(\tilde{T}, \tilde{T}_{a, t_{0}}\right)$ is equivalent to $\left(T, T_{a, t_{0}}\right)$ (i.e., $\left(\tilde{T}, \tilde{T}_{a, t_{0}}\right) \sim$ $\left.\left(T, T_{a, t_{0}}\right)\right)$ with respect to $h: T \rightarrow \tilde{T}$, where $T_{a, t_{0}}=$ $\left\{t \in T: t_{0} \preceq t, \rho\left(t, t_{0}\right)<l\right\}$ is a subset of $T$ and $l>0$ is finite or infinite, depending on $\left(a, t_{0}\right)$;

2) $\tilde{p}\left(h\left(t_{0}\right), a, t_{0}\right)=a$.

We are now in a position to define the hybrid dynamical system.

Definition 3.4 (Hybrid Dynamical System): Let $S$ be a family of motions on $T$, defined as $S \subset\left\{\tilde{p}\left(\cdot, a, t_{0}\right) \in \Lambda\right.$ : $\left.\tilde{p}\left(h\left(t_{0}\right), a, t_{0}\right)=a\right\}$, where $\Lambda=\cup_{\left(a, t_{0}\right) \subset A \times T_{0}}\left\{\tilde{T}_{a, t_{0}} \times\{a\} \times\right.$ $\left.\left\{t_{0}\right\} \rightarrow X\right\}$, and where $h$ is uniquely determined by the specific motion $\tilde{p}\left(\cdot, a, t_{0}\right)$ (as explained in Definition 3.3). Then the five-tuple $\left\{T, X, A, S, T_{0}\right\}$ is called a hybrid dynamical system (HDS).

Remarks-i): In Definition 3.3, a mapping $\tilde{p}\left(\cdot, a, t_{0}\right)$ : $\tilde{T}_{a, t_{0}} \rightarrow X$ is called a motion on $T$, even though this mapping is in fact defined on the subset $\tilde{T}_{a, t_{0}}$ of another time space $\tilde{T}$. This $\tilde{T}$ depends on the initial conditions $\left(a, t_{0}\right)$ and in general varies if $\left(a, t_{0}\right)$ changes. However, any such $\tilde{T}$ is equivalent to the prespecified time space $T$. Due to this equivalence, we may view any motion $\tilde{p}\left(\cdot, a, t_{0}\right): \tilde{T}_{a, t_{0}} \rightarrow X$ as another 
mapping $p\left(\cdot, a, t_{0}\right): T_{a, t_{0}} \rightarrow X$ which is defined on a subset $T_{a, t_{0}}$ of $T$. Accordingly, we can equivalently regard an HDS $\left\{T, X, A, S, T_{0}\right\}$ as a collection of mappings defined only on subsets of $T$.

ii): The preceding way of characterizing motions as mappings that are defined on equivalent but possibly different time spaces is not a redundant exercise and is in fact necessary. This will be demonstrated in Example 2 (Subsection B of the present section).

In the existing literature, several variants for dynamical system definitions are considered (see, e.g., [16]-[19]). Typically, in these definitions time is either $T=R^{+}$or $T=N$, but not both simultaneously, $T_{0}=T$, and depending on the particular definition, various continuity requirements are imposed on the motions which comprise the dynamical system. It is important to note that these system definitions are not general enough to accommodate even the simplest types of hybrid systems, such as, for example, sampled-data systems of the type considered in the example below. In the vast literature on sampled-data systems, the analysis and/or synthesis usually proceeds by replacing the hybrid system by an equivalent system description which is valid only at discrete points in time. This may be followed by a separate investigation to determine what happens to the plant to be controlled between samples.

\section{B. Examples of HDS's}

In the following, we elaborate further on the concepts discussed above by considering two specific examples of HDS's.

Example 1 (Nonlinear Sampled-Data Feedback Control System): We consider systems described by equations of the form

$$
\left\{\begin{array}{l}
\dot{x}(r)=f(x(r))+B u(k), r \in[k, k+1) \\
u(k+1)=C u(k)+D x(k)
\end{array}\right.
$$

where $x \in R^{n}, f \in C^{1}\left[R^{n}, R^{n}\right], f(0)=0, u \in R^{m}$, $B \in R^{n \times m}, C \in R^{m \times m}, D \in R^{m \times n}, r \in R^{+}$, and $k \in N$.

System (1) is an HDS. In the present case the time space is given by

$$
T \triangleq\left\{(r, k) \in R^{2}: r \geq 0, k=[r]\right\} .
$$

The space $T$ is equipped with a metric $\rho$ which has the property that for any $t_{1}=\left(r_{1}, k_{1}\right) \in T$ and $t_{2}=\left(r_{2}, k_{2}\right) \in T$, $\rho\left(t_{1}, t_{2}\right)=\left|r_{1}-r_{2}\right|$. The set $T$ is a completely ordered space in such a way that $t_{1} \prec t_{2}$ if and only if $r_{1}<r_{2}$. The set $T_{0}$ is given by $T_{0}=\left\{(k, k) \in R^{2}: k \in N\right\}$. The motion $p$ determined by (1) is of the form

$$
p(t)=\left[x(r)^{T}, u(k)^{T}\right]^{T}
$$

where in (3) $t=(r, k) \in T$. The state space for system (1) is $X=R^{n} \times R^{m}$ and $A \subset X$.

System (1) may be viewed as an interconnection of two subsystems: a plant which is described by a system of first order ordinary differential equations, and as such, is defined on "continuous-time," $R^{+}$, and a digital controller which is described by a system of first-order ordinary difference

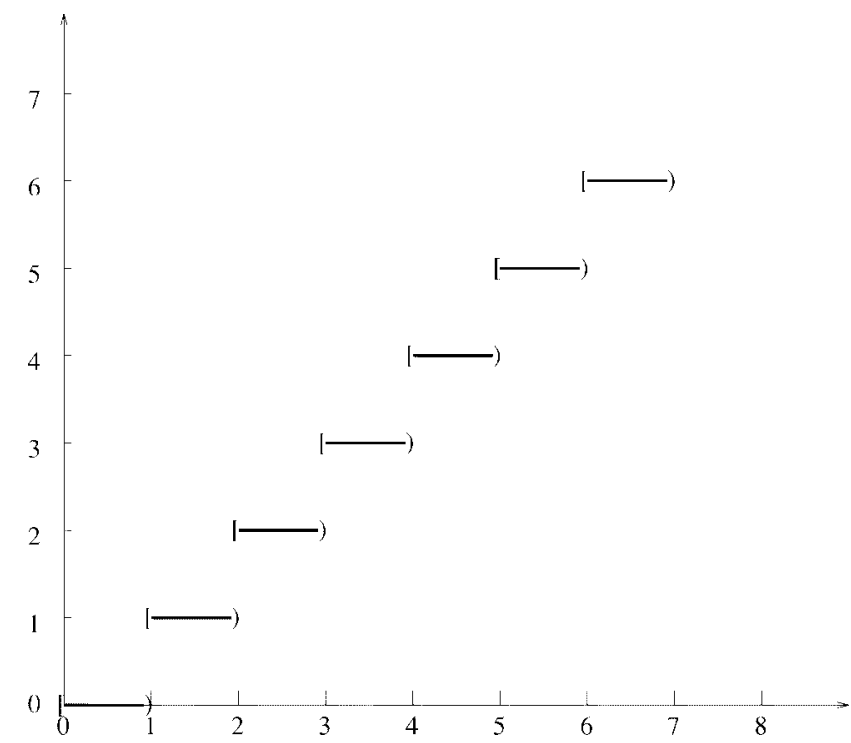

Fig. 1. Graphical representation of the time space $T$ for Example 1.

equations, and as such, is defined on "discrete-time," $N$. The entire system (1) is then defined on $T \subset R^{+} \times N$.

In our considerations of the above sampled-data system, we did not include explicitly a description of the interface between the plant and the digital controller (a sample element) and between the digital controller and the plant (a sample and hold element). In Fig. 1, we provide the "graph" for $T$.

Example 2 (Motion Control System): Several different classes of systems that arise in automation have recently been considered in the literature (see, e.g., [3]). Such systems, which are frequently encountered in the area of motion control, are equipped with certain types of nonlinearities in the form of trigger functions. We consider in the following a special example of such systems which concerns an engine-drive train system for an automobile with an automatic transmission. This system is described by the equations

$$
\left\{\begin{array}{l}
\dot{x}_{1}(r)=x_{2}(r) \\
\dot{x}_{2}(r)=\left[-a\left(x_{2}(r)\right)+u(r)\right] /[1+z([p])] \\
\dot{p}(r)=l\left(x_{1}, x_{2}, u\right),\left(l\left(x_{1}, x_{2}, u\right)>0\right) \\
z([p]+1)=f\left(z([p]), x_{1}\left(r_{[p]}\right), x_{2}\left(r_{[p]}\right)\right)
\end{array}\right.
$$

where $x_{1}, x_{2} \in R$ denote vehicle ground speed and engine rpm, respectively, $u(r) \in R$ denotes the external input as the throttle position, the $a(\cdot)$ term describes the inability of the vehicle to produce torque at high rpms, $z \in Z_{K}$ represents the shift position of the transmission, where $Z_{K}$ is some subset of $N$, and $f: Z_{K} \times R \times R \rightarrow Z_{K}$ determines the shifting rule. The variable $p \in C^{1}\left[R^{+}, R^{+}\right]$represents a special "clock" or "counter." The notation $r_{[p]}$ denotes the most recent time when $p$ passes an integer.

System (4) is an HDS with state space $X=R \times$ $R \times Z_{K}$, time space $T=R^{+}$, and $T_{0}=T$. For any specific initial condition, (4) determines a specific solution $\left[\left(x_{1}(r), x_{2}(r), z([p(r)])\right]\right.$. If we define $\tilde{T}=\left\{(r,[p]) \in R^{2}\right.$ : $r \geq 0, p=p(r)\}$, then $\tilde{T}$ is another time space with metric $\rho$ and order relation $\prec$, having the property that for any $t_{1}=\left(r_{1},\left[p_{1}\right]\right), t_{2}=\left(r_{2},\left[p_{2}\right]\right), \rho\left(t_{1}, t_{2}\right)=\left|r_{1}-r_{2}\right|$, and $t_{1} \prec t_{2}$, if and only if $r_{1}<r_{2}$. The specific solution 


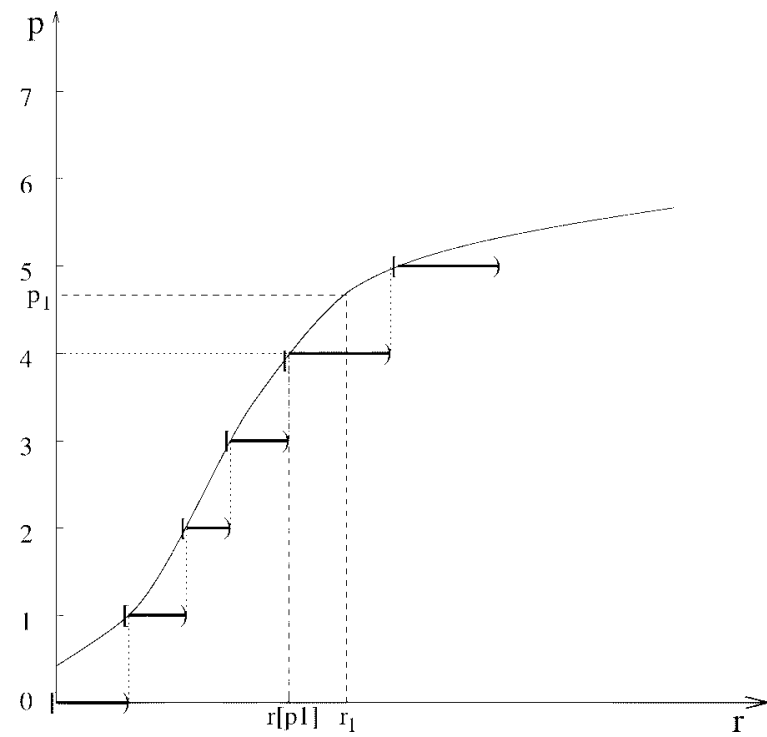

Fig. 2 Graphical representation of a time space $T$ for Example 2.

$\left[\left(x_{1}(r), x_{2}(r), z([p(r)])\right]\right.$ can be regarded as a motion in the form of $q(t)=\left[x_{1}(r), x_{2}(r), z([p])\right]^{T}$, where $t=(r,[p]) \in \tilde{T}$. Although $q(t)$ is a mapping defined on $\tilde{T}$, we still view it as a motion in an HDS defined on $R^{+}$, as defined in Definition 3.4, since $\tilde{T}$ is equivalent to $R^{+}$. In Fig. 3, we depict the graph of the time space $\tilde{T}$ of a specific motion. (We use left brackets to indicate that left end points are included.)

\section{Some Qualitative Characterizations}

In the present paper we will primarily focus our attention on the stability properties of invariant sets of HDS's.

Definition 3.5 (Invariant Set): Let $\left\{T, X, A, S, T_{0}\right\}$ be an HDS. A set $M \subset A$ is said to be invariant with respect to system $S$ if $a \in M$ implies that $p\left(t, a, t_{0}\right) \in M$ for all $t \in T_{a, t_{0}}$, all $t_{0} \in T_{0}$, and all $p\left(\cdot, a, t_{0}\right) \in S$. We will state the above more compactly by saying that $M$ is an invariant set of $S$ or $(S, M)$ is invariant.

Definition 3.6 (Equilibrium): We call $x_{0} \in A$ an equilibrium of an $\operatorname{HDS}\left\{T, X, A, S, T_{0}\right\}$ if the set $\left\{x_{0}\right\}$ is invariant with respect to $S$.

Definition 3.7-Uniform (Asymptotic) Stability: Let $\{T, X$, $\left.A, S, T_{0}\right\}$ be an HDS and let $M \subset A$ be an invariant set of $S$. We say that $(S, M)$ is stable if for every $\epsilon>0$, and $t_{0} \in T_{0}$ there exists a $\delta=\delta\left(\epsilon, t_{0}\right)>0$ such that $d\left(p\left(t, a, t_{0}\right), M\right)<\epsilon$ for all $t \in T_{a, t_{0}}$ and for all $p\left(\cdot, a, t_{0}\right) \epsilon$ $S$, whenever $d(a, M)<\delta$. We say that $(S, M)$ is uniformly stable if $\delta=\delta(\epsilon)$. Furthermore, if $(S, M)$ is stable and if for any $t_{0} \in T_{0}$, there exists an $\eta=\eta\left(t_{0}\right)>0$ such that $\lim _{t \rightarrow \infty} d\left(p\left(t, a, t_{0}\right), M\right)=0$ (i.e., for every $\epsilon>0$, there exits a $t_{\epsilon} \in T$ such that $d\left(p\left(t, a, t_{0}\right), M\right)<\epsilon$ whenever $t \in T$ and $\left.t_{\epsilon} \preceq t\right)$ for all $p\left(\cdot, a, t_{0}\right) \in S$ whenever $d(a, M)<\eta$, then $(S, M)$ is called asymptotically stable. We call $(S, M)$ uniformly asymptotically stable if $(S, M)$ is uniformly stable and if there exits a $\delta>0$ and for every $\epsilon>0$ there exists a $\tau=\tau(\epsilon)>0$ such that $d\left(p\left(t, a, t_{0}\right), M\right)<\epsilon$ for all $t \in\left\{t \in T_{a, t_{0}}: \rho\left(t, t_{0}\right) \geq \tau\right\}$, and all $p\left(\cdot, a, t_{0}\right) \in S$ whenever $d(a, M)<\delta$.

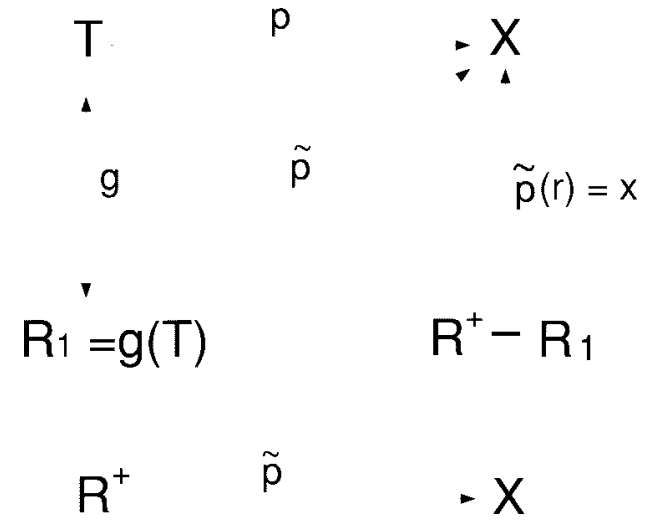

Fig. 3. Representation of the embedding mapping of motions.

Exponential Stability: We call $(S, M)$ exponentially stable if there exists $\alpha>0$, and for every $\epsilon>0$ and $t_{0} \in T_{0}$, there exists a $\delta=\delta(\epsilon)>0$ such that $d\left(p\left(t, a, t_{0}\right), M\right)<\epsilon e^{-\alpha \cdot \rho\left(t, t_{0}\right)}$ for all $t \in T_{a, t_{0}}$ and for all $p\left(\cdot, a, t_{0}\right) \in S$, whenever $d(a, M)<\delta$.

Uniform Boundedness: $S$ is said to be uniformly bounded if for every $\alpha>0$ and for every $t_{0} \in T_{0}$ there exists a $\beta=\beta(\alpha)>0$ (independent of $\left.t_{0}\right)$ such that if $d\left(a, x_{0}\right)<\alpha$, then for all $p\left(\cdot, a, t_{0}\right) \in S d\left(p\left(t, a, t_{0}\right), x_{0}\right)<\beta$ for all $t \in T_{a, t_{0}}$, where $x_{0}$ is an arbitrary point in $X . S$ is uniformly ultimately bounded if there exists $B>0$ and if corresponding to any $\alpha>0$ and $t \in T_{a, t_{0}}$, there exists a $\lambda=\lambda(\alpha)>0$ (independent of $\left.t \in T_{a, t_{0}}\right)$ such that for all $p\left(\cdot, a, t_{0}\right) \in S$, $d\left(p\left(t, a, t_{0}\right), x_{0}\right)<B$ for all $t \in T_{a, t_{0}}$ such that $\rho\left(t, t_{0}\right)>\lambda$, whenever $d\left(a, x_{0}\right)<\alpha$, where $x_{0}$ is an arbitrary point in $X$.

Instability: $(S, M)$ is said to be unstable if $(S, M)$ is not stable.

Remark 3.1: The above definitions of stability, uniform stability, asymptotic stability, uniform asymptotic stability, exponential stability, uniform boundedness, uniform ultimate boundedness, and instability constitute natural adaptations of the corresponding concepts for the usual types of dynamical systems encountered in the literature (refer, e.g., to [16, Secs, 3.1 and 3.2]). In a similar manner as was done above, we can define asymptotic stability in the large, exponential stability in the large, complete instability, and the like, for HDS's of the type considered herein (refer to [22]-[28] and [30]). Due to space limitations, we will not pursue this.

\section{STABILITY OF INVARIANT SETS}

We will accomplish the stability analysis of an invariant set $M$ with respect to an HDS $S$ in two stages. First we embed the HDS $\left\{T, X, A, S, T_{0}\right\}$ (which is defined on a time space $T$ ) into an $\operatorname{HDS}\left\{R^{+}, X, A, \widetilde{S}, R_{0}^{+}\right\}$(which is defined on $R^{+}$). We then show that the stability properties of $(S, M)$ can be deduced from corresponding stability properties of $(\tilde{S}, M)$. Finally, we establish stability results for the HDS $\left\{R^{+}, X, A, \widetilde{S}, R_{0}^{+}\right\}$which is a system with discontinuities in its motions. 


\section{A. Embedding of HDS's into Dynamical} Systems Defined on $R^{+}$

Any time space $T$ (see Definition 3.1) can be embedded into the real space $R^{+}$by means of a mapping $g: T \rightarrow R^{+}$ having the following properties: 1) $g\left(t_{\min }\right)=0$, where $t_{\min }$ denotes the minimum element in $T$ and 2) $g(t)=\rho\left(t, t_{\min }\right)$ for $t \neq t_{\text {min }}$. Note that if we let $R_{1}=g(T)$, then $g$ is an isometric mapping from $T$ to $R_{1}$ [i.e., $g$ is a bijection from $T$ onto $R_{1}$, and for any $t_{1}, t_{2} \in T$ such that $t_{1} \prec t_{2}$ it is true that $\left.\rho\left(t_{1}, t_{2}\right)=g\left(t_{2}\right)-g\left(t_{1}\right)\right]$.

The above embedding mapping gives rise to the following concepts.

Definition 4.1 (Embedding of a Motion ): Let $\quad\{T, X, A$, $\left.S, T_{0}\right\}$ be an HDS, let $x \in A$ be fixed, and let $g: T \rightarrow R^{+}$ be the embedding mapping defined above. Suppose that $p\left(\cdot, a, t_{0}\right) \in S$ is a motion defined on $T_{a, t_{0}}$. Let $\tilde{p}\left(\cdot, a, r_{0}\right)$ : $R_{r_{0}}^{+} \rightarrow X$, where $R_{r_{0}}^{+}=\left\{r \in R^{+}: r \geq r_{0}\right\}$, be a function having the following properties: 1) $r_{0}=g\left(t_{0}\right)$; 2) $\tilde{p}\left(r, a, r_{0}\right)=p\left(g^{-1}(r), a, t_{0}\right)$ if $r \in R_{1}=g(T)$; and 3) $\tilde{p}\left(r, a, r_{0}\right)=x$ if $r \notin R_{1}$. We call $\tilde{p}\left(\cdot, a, r_{0}\right)$ the embedding of $p\left(\cdot, a, t_{0}\right)$ from $T$ to $R^{+}$with respect to $x$. The graphic interpretation of this embedding is given in Fig. 3.

It turns out that $\tilde{p}\left(\cdot, a_{0}, r_{0}\right)$ is a motion for another dynamical system which we define next.

Definition 4.2 (Embedding of an HDS ): Let $\{T, X, A$, $\left.S, T_{0}\right\}$ be an HDS and let $x \in A$. The HDS $\left\{R^{+}, X, A, \widetilde{S}, R_{0}^{+}\right\}$ is called the embedding of $\left\{T, X, A, S, T_{0}\right\}$ from $T$ to $R^{+}$with respect to (w.r.t.) $x$, where $R_{0}^{+}=g\left(T_{0}\right)$ and $\tilde{S} \triangleq\left\{\tilde{p}\left(\cdot, a_{0}, r_{0}\right): \tilde{p}\left(\cdot, a_{0}, r_{0}\right)\right.$ is the embedding of $p\left(\cdot, a_{0}, t_{0}\right)$ w.r.t. $\left.x, p\left(\cdot, a_{0}, t_{0}\right) \in S\right\}$.

In general, different choices of $x$ will result in different embeddings of an HDS. It is important to note, however, that different embeddings corresponding to different elements $x$ contained in the same invariant set $M$ will possess identical stability properties.

In view of the above definitions and observations, any HDS defined on an abstract time space $T$ can be embedded into another HDS defined on real time space $R^{+}$. The latter system consists of motions which in general may be discontinuous and has similar qualitative properties as the original hybrid system defined on an abstract time space. This is summarized in the next result.

Proposition 4.1: Suppose $\left\{T, X, A, S, T_{0}\right\}$ is an HDS. Let $M \subset A$ be an invariant subset of $S$, and let $x$ be any fixed point in $M$. Let $\left\{R^{+}, X, A, \tilde{S}, R_{0}^{+}\right\}$be the embedding of $\left\{T, X, A, S, T_{0}\right\}$ from $T$ to $R^{+}$with respect to $x$. Then $M$ is also an invariant subset of system $\tilde{S}$ and $(S, M)$ and $(\tilde{S}, M)$ possess identical stability properties.

Proof: By construction it is clear that $M$ is invariant with respect to $S$ if and only if $M$ is invariant with respect to $\tilde{S}$. In the following, we show in detail that $(S, M)$ is uniformly asymptotically stable if and only if $(\tilde{S}, M)$ is uniformly asymptotically stable. The equivalence of the other qualitative properties between $(\widetilde{S}, M)$ and $(S, M)$, such as stability, exponential stability, uniform boundedness, and uniform ultimate boundedness, can be established in a similar manner (see [22]-[28] and [30]) and will therefore not be presented here.
Our proof consists of two parts. First, we show that $(S, M)$ is uniformly stable if and only if $(\tilde{S}, M)$ is uniformly stable. Next, we show that $(S, M)$ is uniformly asymptotically stable if and only if $(\tilde{S}, M)$ is uniformly asymptotically stable.

1): If $(S, M)$ is uniformly stable, we know that for every $\epsilon>0$ there exists a $\delta(\epsilon)>0$ such that for every $t_{0} \in T_{0}$, $d\left(p\left(t, a, t_{0}\right), M\right)<\epsilon$ for all $a \in A$ with $d(a, M)<\delta$, for all $t \in T_{a, t_{0}}$ and all $p\left(t, a, t_{0}\right) \in S$. For any $\tilde{p}\left(r, a, r_{0}\right) \in \tilde{S}$ it is true that

$$
\begin{aligned}
& d\left(\tilde{p}\left(r, a, r_{0}\right), M\right) \\
& \quad=\left\{\begin{array}{cc}
0, & \text { if } r \notin R_{1} \\
d\left(p\left(g^{-1}(r), a, g^{-1}\left(r_{0}\right)\right), M\right), & \text { if } r \in R_{1}
\end{array}\right.
\end{aligned}
$$

where $r \in R^{+}$, and $r_{0} \in R_{0}^{+}$. Hence, whenever $d(a, M)<\delta$ is satisfied, we have either $d\left(\tilde{p}\left(r, a, r_{0}\right), M\right)=0$ for $r \notin R_{1}$ or $d\left(\tilde{p}\left(r, a, r_{0}\right), M\right)=d\left(p\left(g^{-1}(r), a, g^{-1}\left(r_{0}\right)\right), M\right)<\epsilon$ for $r \in R_{1}$. This leads to the conclusion that $(\tilde{S}, M)$ is uniformly stable.

Next, assume that $(\tilde{S}, M)$ is uniformly stable. Then for every $\epsilon>0$ there exists a $\delta(\epsilon)>0$ such that for every $r_{0} \in R_{0}^{+}, d\left(\tilde{p}\left(r, a, r_{0}\right), M\right)<\epsilon$ for all $a \in A$ with $d(a, M)<$ $\delta$, for all $r_{0} \in R_{0}^{+}$and all $\tilde{p}\left(\cdot, a, t_{0}\right) \in \widetilde{S}$. Therefore, for any $p\left(t, a, t_{0}\right) \in S$ satisfying $d(a, M)<\delta$, it follows that $d\left(p\left(t, a, t_{0}\right), M\right)=d\left(\tilde{p}\left(g(t), a, g\left(t_{0}\right)\right), M\right)<\epsilon$. We conclude that $(S, M)$ is uniformly stable.

2): If $(S, M)$ is uniformly asymptotically stable, we know that $(S, M)$ is uniformly stable, and there exists a $\delta>0$ and for every $\epsilon>0$ there exists a $\tau=\tau(\epsilon)>0$ such that $d\left(p\left(t, a, t_{0}\right), M\right)<\epsilon$ for all $t \in T, t_{0} \prec t, \rho\left(t, t_{0}\right) \geq \tau$ and all $p\left(t, a, t_{0}\right) \in S$ whenever $d(a, M)<\delta$, where $a \in A$, and $t_{0} \in$ $T_{0}$. For any $\tilde{p}\left(r, a, r_{0}\right) \in \widehat{S}$ satisfying $d(a, M)<\delta$, it is true that $d\left(\tilde{p}\left(r, a, r_{0}\right), M\right)=0$ for $r \notin R_{1}$ or $d\left(\tilde{p}\left(r, a, r_{0}\right), M\right)=$ $d\left(p\left(g^{-1}(r), a, g^{-1}\left(r_{0}\right)\right), M\right)$ if $r \in R_{1}$. Furthermore, since in the latter case it is true that $r-r_{0}=\rho\left(g^{-1}(r), g^{-1}\left(r_{0}\right)\right)$, it follows that $d\left(\tilde{p}\left(r, a, r_{0}\right), M\right)<\epsilon$ as long as $r-r_{0}>\tau$. Therefore, by using the conclusions of part 1), we conclude that $(\tilde{S}, M)$ is uniformly asymptotically stable.

If $(\tilde{S}, M)$ is uniformly asymptotically stable, we know that $(\tilde{S}, M)$ is uniformly stable and there exists a $\delta>0$ and for every $\epsilon>0$ there exists a $\tau=\tau(\epsilon)>0$ such that $d\left(\tilde{p}\left(r, a, r_{0}\right), M\right)<\epsilon$ for all $r-r_{0}>\tau$ and all $\tilde{p}\left(r, a, r_{0}\right) \in \tilde{S}$ whenever $d(a, M)<\delta$, where $a \in A$, and $r_{0} \in R_{0}^{+}$. Therefore, for any $p\left(t, a, t_{0}\right) \in S$ satisfying $d(a, M)<\delta$, it is true that $d\left(p\left(t, a, t_{0}\right), M\right)=d\left(\tilde{p}\left(g(t), a, g\left(t_{0}\right)\right), M\right)<\epsilon$ for all $t \in T, t_{0} \prec t, \rho\left(t_{0}, t\right)>\tau$ since $\rho\left(t_{0}, t\right)=g(t)-$ $g\left(t_{0}\right)$. Therefore, we conclude that $(S, M)$ is also uniformly asymptotically stable.

In view of Proposition 4.1 and other similar results [22]-[28], [30], the qualitative properties (such as the stability properties of an invariant set) of an HDS $S$ can be deduced from the corresponding properties of the dynamical system $\tilde{S}$, defined on $R^{+}$, into which system $S$ has been embedded. Although dynamical systems which are defined on $R^{+}$have been studied extensively (refer, e.g., to [16]-[19]), it is usually assumed in these works that the motions are continuous, and as such the results in these works are not directly applicable in the analysis of the dynamical system $\left\{R^{+}, X, A, \widetilde{S}, R_{0}^{+}\right\}$. 


\section{B. Lyapunov Stability Results}

In the following, we establish some stability results for HDS's $\left\{R^{+}, X, A, \tilde{S}, R_{0}^{+}\right\}$with discontinuous motions $\tilde{p}$. To simplify our notation, we will henceforth drop the tilde, $\sim$, from $\tilde{S}$ and $\tilde{p}$ and simply write $\left\{R^{+}, X, A, S, R_{0}^{+}\right\}$in place of $\left\{R^{+}, X, A, \tilde{S}, R_{0}^{+}\right\}$.

Theorem 4.1 (Lyapunov Stability): Let $\left\{R^{+}, X, A, S, R_{0}^{+}\right\}$ be an HDS, and let $M \subset A$. Assume that there exists a $V: X \times R^{+} \rightarrow R^{+}$and $\phi_{1}, \phi_{2} \in K$ defined on $R^{+}$such that

$$
\phi_{1}(d(x, M)) \leq V(x, r) \leq \phi_{2}(d(x, M))
$$

for all $x \in X, r \in R^{+}$.

1): Assume that for any $p\left(\cdot, a, r_{0}\right) \in S, V\left(p\left(r, a, r_{0}\right), r\right)$ is continuous everywhere on $R_{r_{0}}^{+} \triangleq\left\{r \in R^{+}: r \geq r_{0}\right\}$ except on an unbounded closed discrete subset $E$ of $R_{r_{0}}^{+}$ ( $E$ depends on $p$ ). Also, assume that if we denote $E=$ $\left\{r_{1}, r_{2}, \cdots\right\}$, then $V\left(p\left(r_{n}, a, r_{0}\right), r_{n}\right)$ is nonincreasing for $n=$ $0,1, \cdots$. Furthermore, assume that there exists $f \in C\left[R^{+}, R^{+}\right]$ independent of $p \in S$ such that $f(0)=0$ and such that $V\left(p\left(r, a, r_{0}\right), r\right) \leq f\left(V\left(p\left(r_{n}, a, r_{0}\right), r_{n}\right)\right)$ for $r \in\left(r_{n}, r_{n+1}\right)$, $n=0,1, \cdots$. Then $(S, M)$ is invariant and uniformly stable .

2 ): If in addition to the assumptions given in 1) there exists $\phi_{3} \in K$ defined on $R^{+}$, such that $D V\left(p\left(r_{n}, a, r_{0}\right), r_{n}\right) \leq$ $-\phi_{3}\left(d\left(p\left(r_{n}, a, r_{0}\right), M\right)\right)$, where $D V\left(p\left(r_{n}, a, r_{0}\right), r_{n}\right) \triangleq$ $\frac{1}{r_{n+1}-r_{n}}\left[V\left(p\left(r_{n+1}, a, r_{0}\right), r_{n+1}\right)-V\left(p\left(r_{n}, a, r_{0}\right), r_{n}\right)\right]$, then $(S, M)$ is uniformly asymptotically stable.

Proof 1): We first prove that $(S, M)$ is invariant. If $a \in$ $M$, then $V\left(p\left(r_{0}, a, r_{0}\right), r_{0}\right)=0$ since $V\left(p\left(r_{0}, a, r_{0}\right), r_{0}\right)=$ $V\left(a, r_{0}\right) \leq \phi_{2}(d(a, M))=0$ and $d(a, M)=0$. Therefore, we know that $V\left(p\left(r_{n}, a, r_{0}\right), r_{n}\right)=0$ for all $n \geq 0$, and furthermore $V\left(p\left(r, a, r_{0}\right), r\right)=0$ for all $r \geq r_{0}$ since $V\left(p\left(r, a, r_{0}\right), r\right) \leq f\left(V\left(p\left(r_{n}, a, r_{0}\right), r_{n}\right)\right)$. It is then implied that $p\left(r, a, r_{0}\right) \in M$ for all $r \geq r_{0}$. Therefore, $(S, M)$ is invariant by definition.

Since $f$ is continuous and $f(0)=0$, then for any $\epsilon>0$ there exists $\delta=\delta(\epsilon)>0$ such that $f(y)<\phi_{1}(\epsilon)$ as long as $0 \leq$ $y<\delta$. We can assume that $\delta \leq \phi_{1}(\epsilon)$. Thus for any motion $p\left(\cdot, a, r_{0}\right) \in S$, as long as the initial condition $d(a, M)<$ $\phi_{2}^{-1}(\delta)$ is satisfied, then $V\left(p\left(r_{0}, a, r_{0}\right), r_{0}\right)=V\left(a, r_{0}\right) \leq$ $\phi_{2}(d(a, M))<\phi_{2}\left(\phi_{2}^{-1}(\delta)\right)=\delta$ and $V\left(p\left(r_{n}, a, r_{0}\right), r_{n}\right)<\delta$ for $n=1,2, \cdots$, since $V\left(p\left(r_{n}, a, r_{0}\right), r_{n}\right)$ is nonincreasing. Furthermore, for any $r \in\left(r_{n}, r_{n+1}\right)$ we can conclude that $V\left(p\left(r, a, r_{0}\right), r\right) \leq f\left(V\left(p\left(r_{n}, a, r_{0}\right), r_{n}\right)\right)<\phi_{1}(\epsilon)$ and $d\left(p\left(r, a, r_{0}\right), M\right) \leq \bar{\phi}_{1}^{-1}\left(V\left(p\left(r, a, r_{0}\right), r\right)\right)<\phi_{1}^{-1}\left(\phi_{1}(\epsilon)\right)$. Therefore, by definition, $(S, M)$ is uniformly stable.

$2)$ : Letting $z_{n}=V\left(p\left(r_{n}, a, r_{0}\right), r_{n}\right)$, we obtain from the assumptions of the theorem that $z_{n+1}-z_{n} \leq-\phi_{3}$ o $\phi_{2}^{-1}\left(z_{n}\right)\left(r_{n+1}-r_{n}\right)$ for $n=0,1, \cdots$, . If we denote $\phi=$ $\phi_{3} \circ \phi_{2}^{-1}$, then $\phi \in K$ and the above inequality becomes $z_{n+1}-z_{n} \leq-\phi\left(z_{n}\right)\left(r_{n+1}-r_{n}\right)$. Since $\left\{z_{n}\right\}$ is nonincreasing and $\phi_{3} \in K$, it follows that $z_{k+1}-z_{k} \leq-\phi\left(z_{k}\right)\left(r_{k+1}-r_{k}\right) \leq$ $-\phi\left(z_{n}\right)\left(r_{k+1}-r_{k}\right)$ for all $k \leq n$. We thus obtain that $z_{n+1}-z_{0} \leq-\phi\left(z_{n}\right)\left(r_{n+1}-r_{0}\right)$, for all $n \geq 0$. It follows that

$$
\phi\left(z_{n}\right) \leq \frac{z_{0}-z_{n+1}}{r_{n+1}-r_{0}} \leq \frac{z_{0}}{r_{n+1}-r_{0}} .
$$

Now consider a fixed $\delta>0$. For any given $\epsilon>0$, we can choose a $\tau>0$ such that

$\max \left\{\phi_{1}^{-1}\left(\phi^{-1}\left(\frac{\phi_{2}(\delta)}{\tau}\right)\right), \phi_{1}^{-1}\left(f\left(\phi^{-1}\left(\frac{\phi_{2}(\delta)}{\tau}\right)\right)\right)\right\}<\epsilon$

since $\phi_{1}, \phi_{2}, \phi \in K$ and $f(0)=0$. For any $a \in A$ with $d(a, M)<\delta$ and any $r_{0} \in R_{0}^{+}$, we are now able to show that $d\left(p\left(r, a, r_{0}\right), M\right)<\epsilon$ whenever $r \geq r_{0}+\tau$. The above statement is true because for any $r \geq r_{0}+\tau, r$ must belong to some interval $\left[r_{n}, r_{n+1}\right)$ for some $n \in N$, i.e., $r \in\left[r_{n}, r_{n+1}\right)$. Therefore, we know that $r_{n+1}-r_{0}>\tau$. It follows from (5) that $\phi\left(z_{n}\right) \leq \frac{\phi_{2}(\delta)}{\tau}$, which implies that

$$
V\left(p\left(r_{n}, a, r_{0}\right), r_{n}\right)=z_{n} \leq \phi^{-1}\left(\frac{\phi_{2}(\delta)}{\tau}\right)
$$

and

$$
V\left(p\left(r, a, r_{0}\right), r_{0}\right) \leq f\left(\phi^{-1}\left(\frac{\phi_{2}(\delta)}{\tau}\right)\right)
$$

if $r \in\left(r_{n}, r_{n+1}\right)$. In the case when $r=r_{n}$, it follows from (7) that $d\left(p\left(r_{n}, a, r_{0}\right), M\right)<\epsilon$, noticing that (6) holds. In the case when $r \in\left(r_{n}, r_{n+1}\right)$, we can conclude from (7) that $d\left(p\left(r, a, r_{0}\right), M\right)<\epsilon$. This proves that $(S, M)$ is uniformly asymptotically stable.

Remarks 1): In Theorem 4.1 (and in several subsequent results) we required that every motion be continuous everywhere except on an unbounded closed discrete set $E=\left\{r_{1}, r_{2}, \cdots\right\}$. With this requirement, we ensure that $r_{n}$ will converge to $\infty$ without finite accumulation. The reason for requiring this is because our main interest concerns the asymptotic behavior (when $r$ goes to $\infty$ ) of the (discontinuous) motions of HDS's.

2 ): In cases where the qualitative behavior of a dynamical system is of interest when time approaches some finite instance (point), say $r_{\infty}<\infty$, no essential difficulties are encountered in establishing qualitative results similar to those given above. In this case we require that each motion be continuous everywhere on $\left\{r: r<r_{\infty}\right\}$, except on $E=\left\{r_{1}, r_{2}, \cdots\right\}$ with $\left\{r_{n}\right\} \rightarrow r_{\infty}$.

In the following we state additional Lyapunov stability results for HDS's. We omit the proofs of these results due to space limitations. For some of these proofs, refer to [22]-[28] and [30].

Theorem 4.2 (Exponential Stability): Let $\quad\left\{R^{+}, X, A\right.$, $\left.S, R_{0}^{+}\right\}$be an HDS, and let $M \subset A$. Assume that there exists a function $V: X \times R^{+} \rightarrow R^{+}$and four positive constants $a_{1}, a_{2}, a_{3}$, and $b$ such that, $a_{1}[d(x, M)]^{b} \leq V(x, r)$ $\leq a_{2}[d(x, M)]^{b}$ for all $x \in X, r \in R$. Assume that for any $p\left(\cdot, a, r_{0}\right) \in S, V\left(p\left(r, a, r_{0}\right), r\right)$ is continuous everywhere on $R_{r_{0}}^{+} \triangleq\left\{r \in R^{+}: r \geq r_{0}\right\}$ except on an unbounded closed discrete subset $E$ of $R_{r_{0}}^{+}$. Let $E=\left\{r_{1}, r_{2}, \cdots\right\}$ with $r_{n}$ strictly increasing. Furthermore, assume that there exists $f \in C\left[R^{+}, R^{+}\right]$such that $V\left(p\left(r, a, r_{0}\right), r\right) \leq f\left(V\left(p\left(r_{n}, a, r_{0}\right), r_{n}\right)\right)$ for $r \in\left(r_{n}, r_{n+1}\right)$, $n=0,1, \cdots$, and that for some positive constant $q, f$ satisfies $f(t)=o\left(|t|^{q}\right) \quad$ as $\quad|t| \rightarrow 0$ (i.e., $\lim _{t \rightarrow 0} \frac{f(t)}{|t|^{q}}=0$ ). Assume that $D V\left(p\left(r_{n}, a, r_{0}\right), r_{n}\right) \leq-a_{3}\left[V\left(p\left(r_{n}, a, r_{0}\right), r_{n}\right)\right]^{b}$, for all $n$. Then $(S, M)$ is exponentially stable in the large ( $D V$ is defined in Theorem 4.1). 
Theorem 4.3 (Boundedness): Let $\left\{R^{+}, X, A, S, R_{0}^{+}\right\}$be an HDS, and let $M \subset A$ where $M$ is bounded. Assume that there exists a function $V: X \times R^{+} \rightarrow R^{+}$and $\phi_{1} \in K R, \phi_{2} \in K R$ such that $\phi_{1}(d(x, M)) \leq V(x, r) \leq \phi_{2}(d(x, M))$ for all $x \in X$ and $r \in R^{+}$.

1): Assume that for any $p\left(\cdot, a, r_{0}\right) \in S, V\left(p\left(r, a, r_{0}\right), r\right)$ is continuous everywhere on $R_{r_{0}}^{+} \triangleq\left\{r \in R^{+}: r \geq r_{0}\right\}$ except on an unbounded closed discrete subset $E$ of $R_{r_{0}}^{+}$. Let $E=\left\{r_{1}, r_{2}, \cdots\right\}$ with $r_{n}$ strictly increasing. Assume that $V\left(p\left(r_{n}, a, r_{0}\right), r_{n}\right)$ is nonincreasing for all $r_{n}$ such that $d\left(p\left(r_{n}, a, r_{0}\right), M\right) \geq \xi$, where $\xi$ is a constant. Furthermore, assume that there exists $f \in C\left[R^{+}, R^{+}\right]$ such that $V\left(p\left(r, a, r_{0}\right), r\right) \leq f\left(V\left(p\left(r_{n}, a, r_{0}\right), r_{n}\right)\right)$ for $r \in$ $\left(r_{n}, r_{n+1}\right), n=0,1, \cdots$, and that there exists $\zeta>0$ such that $d\left(p\left(r_{n+1}, a, r_{0}\right), M\right) \leq \zeta$ whenever $d\left(p\left(r_{n}, a, r_{0}\right), M\right) \geq \xi$. Then $S$ is uniformly bounded.

2): In part 1), assume in addition that there exists $\phi_{3} \in K$ defined on $R^{+}$such that

$$
D V\left(p\left(r_{n}, a, r_{0}\right), r_{n}\right) \leq-\phi_{3}\left(d\left(p\left(r_{n}, a, r_{0}\right), M\right)\right)
$$

for all $r_{n}$ such that $d\left(p\left(r_{n}, a, r_{0}\right), M\right) \geq \xi$. Then $S$ is uniformly ultimately bounded.

Theorem 4.4 (Instability): Let $\left\{R^{+}, X, A, S, R_{0}^{+}\right\}$be an HDS, and let $M \subset A$. Assume that there exists a function $V: X \times R^{+} \rightarrow R^{+}$which satisfies the following conditions.

1): There exists a $\phi \in K$ defined on $R^{+}$such that $V(x, r) \leq \phi(d(x, M))$.

2): For any $p\left(\cdot, a, r_{0}\right) \in S, V\left(p\left(r, a, r_{0}\right), r\right)$ is continuous everywhere on $R_{r_{0}}^{+} \triangleq\left\{r \in R^{+}: r \geq r_{0}\right\}$ except on an unbounded closed discrete subset $E$ of $R_{r_{0}}^{+}$, and there exists $\psi \in$ $K$ such that $D V\left(p\left(r_{n}, a, r_{0}\right), r_{n}\right) \geq \psi\left(d\left(p\left(r_{n}, a, r_{0}\right), M\right)\right)$ for all $n$.

3): In every neighborhood of $M$ there are points $x$ such that $V\left(x, r_{0}\right)>0$. Then $M$ is unstable w.r.t. $S$.

For further results which are in the spirit of the above theorems, refer to [24] and [28].

\section{Converse Theorems}

In this subsection we establish a converse to Theorem 4.1 for the case of uniform stability and uniform asymptotic stability under some additional mild assumptions. We will be concerned with the special cases when $R_{0}^{+}=R^{+}$ and $T_{0}=T$. Accordingly, we will simplify our notation by writing $\left\{R^{+}, X, A, S\right\}$ and $\{T, X, A, S\}$ in place of $\left\{R^{+}, X, A, S, R_{0}^{+}\right\}$and $\left\{T, X, A, S, T_{0}\right\}$.

Assumption 4.1: Let $\left\{R^{+}, X, A, S\right\}$ be an HDS. Assume that: 1) for any $p\left(\cdot, a, r_{0}\right) \in S$, there exists a $\tilde{p}\left(\cdot, a_{1}, r_{1}\right) \in S$ with $r_{1} \geq r_{0}, a_{1}=p\left(r_{1}, a, r_{0}\right)$ such that $\tilde{p}\left(r, a_{1}, r_{1}\right)=$ $p\left(r, a, r_{0}\right)$ for all $r \geq r_{1}$ and 2) for any two motions $p_{i}\left(\cdot, a_{i}, r_{i}\right) \in S, i=1,2$, if $a_{2}=p_{1}\left(r_{2}, a_{1}, r_{1}\right)$, then there exists a $\hat{p}\left(\cdot, a_{1}, r_{1}\right) \in S$ such that $\hat{p}\left(r, a_{1}, r_{1}\right)=p_{1}\left(r, a_{1}, r_{1}\right)$ for $r \in\left[r_{1}, r_{2}\right)$ and $\hat{p}\left(r, a_{1}, r_{1}\right)=p_{2}\left(r, a_{2}, r_{2}\right)$ for $r \geq r_{2}$. $\square$

The above assumption is also utilized in the analysis of continuous dynamical systems (see, e.g., [16, Assumption 4.5.1]). In this assumption, we may view $\tilde{p}\left(\cdot, a_{1}, r_{1}\right)$ in 1) as a partial motion of the motion $p\left(\cdot, a, r_{0}\right)$, and we may view $\hat{p}\left(\cdot, a_{1}, r_{1}\right)$ in 2$)$ as a composition of $p_{1}\left(\cdot, a_{1}, r_{1}\right)$ and $p_{2}\left(\cdot, a_{2}, r_{2}\right)$. With this convention, Assumption 4.1 can be restated in the following manner: 1) any partial motion is a motion in $S$ and 2) any composition of two motions is a motion in $S$.

Theorem 4.5: Let $\left\{R^{+}, X, A, S\right\}$ be an HDS and let $M \subset$ $A$ be an invariant set, where $A$ is assumed to be a neighborhood of $M$. Suppose that $S$ satisfies Assumption 4.1 and that $(S, M)$ is uniformly stable. Then there exist neighborhoods $A_{1}, X_{1}$ such that $A_{1} \subset X_{1} \subset A$, and a mapping $V$ : $X_{1} \times R^{+} \rightarrow R^{+}$which satisfies the following conditions: 1$)$ there exists $\phi_{1}, \phi_{2} \in K$ such that $\phi_{1}(d(x, M)) \leq V(x, r) \leq$ $\phi_{2}(d(x, M))$ for all $(x, r) \in X_{1} \times R^{+}$and 2$)$ for every $p\left(\cdot, a, r_{0}\right) \in S$ with $a \in A_{1}, V\left(p\left(r, a, r_{0}\right), r\right)$ is nonincreasing for all $r \geq r_{0}$.

The proof of Theorem 4.5 follows along the same lines as the proof of an existing converse result for the uniform stability of continuous dynamical systems. This proof, however, does not make use of any continuity assumptions for the dynamical system (refer to the proof of [16, Th. 4.5.2]). For the converse theorem of uniform asymptotic stability, the results in the literature cannot be adopted directly because of continuity assumptions in the proofs of those results (see, e.g., [16]). However, under some additional mild assumptions, we will be able to establish a converse theorem for the uniform asymptotic stability of invariant sets of the types of hybrid systems considered herein.

Assumption 4.2: Let $\left\{R^{+}, X, A, S\right\}$ be an HDS defined on $R^{+}$and assume that every $p\left(\cdot, a, r_{0}\right) \in S$ is continuous everywhere on $R^{+}$except possibly on $E=\left\{r_{1}, r_{2}, \cdots\right\}$ [where $E$ depends on $\left(a, r_{0}\right)$ ], and that: 1$) l \triangleq \inf \left\{r_{n+1}-r_{n}\right.$ : $\left.\left(a, r_{0}\right) \in A \times R^{+}, n=1,2, \cdots\right\}>0$ and 2) $L \triangleq \sup \left\{r_{n+1}-\right.$ $\left.r_{n}:\left(a, r_{0}\right) \in A \times R^{+}, n=0,1, \cdots\right\}<\infty$.

Remark: Notice that in part 2) of Assumption 4.2, $n$ starts from zero. However in part 1), we require only that $n$ starts from one since in general there is no lower limit for $r_{1}-r_{0}$.

We are now in a position to state and prove the following converse result.

Theorem 4.6: Let $\left\{R^{+}, X, A, S\right\}$ be an HDS and let $M \subset$ $A$ be an invariant set. Assume that $S$ satisfies Assumptions 4.1 and 4.2 , and furthermore assume that for every $\left(a, r_{0}\right) \in$ $A \times R^{+}$, there exists a unique $p\left(\cdot, a, t_{0}\right) \in S$. Let $(S, M)$ be uniformly asymptotically stable. Then, there exist neighborhoods $A_{1}$ and $X_{1}$ of $M$ such that $A_{1} \subset X_{1} \subset A$, and a mapping $V: X_{1} \times R^{+} \rightarrow R^{+}$which satisfies the following conditions.

1) There exist $\phi_{1}, \phi_{2} \in K$ such that $\phi_{1}(d(x, M)) \leq$ $V(x, r) \leq \phi_{2}(d(x, M))$ for all $(x, r) \in X_{1} \times R^{+}$.

2) There exists $\phi_{3} \in K$ such that for all $p\left(\cdot, a, r_{0}\right) \in S$, we have $D V\left(p\left(r_{n}, a, r_{0}\right), r_{n}\right) \leq$ - $\phi_{3}\left(d\left(p\left(r_{n}, a, r_{0}\right), M\right)\right)$ where $a \in A_{1}, n=0,1, \cdots$, and where $D V$ is defined in Theorem 4.1

3) There exists an $f \in C\left(R^{+}, R^{+}\right), f(0)=0$, such that $V\left(p\left(r, a, r_{0}\right), r\right) \leq f\left(V\left(p\left(r_{n}, a, r_{0}\right), r_{n}\right)\right.$ for all $r \in\left[r_{n}, r_{n+1}\right)$ and all $a \in A_{1}, r_{0} \in R^{+}$.

In the proof of the above theorem, we will require the following preliminary result. 
Lemma 4.1: Let $\beta \in L$ be defined on $R^{+}$. Then there exists a function $\alpha \in K$ defined on $[0, \beta(0)]$ such that for any closed discrete subset $\left\{r_{0}, r_{1}, \cdots\right\} \subset R^{+}$satisfying $\inf \left\{r_{n+1}-r_{n}\right.$ : $n=1,2 \cdots\}>0$, it is true that $\sum_{i=0}^{\infty} \alpha\left(\beta\left(r_{i}-r_{0}\right)\right)<+\infty$.

Proof of Lemma 4.1: We define $\eta \in C\left[R_{+}, R_{+}\right]$, where $R_{+}=(0, \infty)$, by

$$
\eta(t)=\left\{\begin{array}{l}
\beta(t) / t, t \in(0,1) \\
\beta(t), t \in[1, \infty) .
\end{array}\right.
$$

Clearly, $\eta(t)$ is strictly decreasing for all $t>0$, $\lim _{t \rightarrow 0^{+}} \eta(t)=+\infty$ and $\eta(t) \geq \beta(t)$ for all $t>0$. Furthermore, $\eta$ is invertible, $\eta^{-1}$ is strictly decreasing, and $\eta^{-1}(\beta(\tau)) \geq \eta^{-1}(\eta(\tau))=\tau$ for all $\tau>0$.

We now define $\alpha(0)=0$ and $\alpha(r)=\exp \left(-\eta^{-1}(r)\right)$ for $r>0$. Then it is obvious that $\alpha \in K$ and $\alpha(\beta(\tau))=$ $\exp \left(-\eta^{-1}(\beta(\tau))\right) \leq \exp (-\tau)$. It follows that $\sum_{i=0}^{\infty} \alpha\left(\beta\left(r_{i}-\right.\right.$ $\left.\left.r_{0}\right)\right) \leq \sum_{i=0}^{\infty} \exp \left(r_{0}-r_{i}\right) \leq 1+\sum_{i=1}^{\infty} \exp \left(r_{0}-r_{i}\right) \leq$ $1+\sum_{i=1}^{\infty} \exp \left(r_{1}-r_{i}\right)$.

If we denote $l=\inf \left\{r_{n+1}-r_{n}: n=1,2 \cdots\right\}$, we know that $r_{i}-r_{1} \geq(i-1) l$. Hence it is true that

$$
\begin{aligned}
& \sum_{i=0}^{\infty} \alpha\left(\beta\left(r_{i}-r_{0}\right)\right) \leq 1+\sum_{i=1}^{\infty} \exp (-(i-1) l) \\
& =1+\frac{1}{1-\exp (-l)}<+\infty
\end{aligned}
$$

We now proceed to the proof of Theorem 4.6.

Proof of Theorem 4.6: Since $(S, M)$ is uniformly asymptotically stable, we know by Theorem 4.5 that there exist some neighborhoods $\tilde{A}_{1}$ and $\tilde{X}_{1}$ of $M$ such that $\tilde{A}_{1} \subset \tilde{X}_{1} \subset A$ and a mapping $\tilde{V}: \tilde{X}_{1} \times R^{+} \rightarrow R^{+}$which satisfies the following conditions.

1) There exist $\widetilde{\phi_{1}}, \widetilde{\phi}_{2} \in K$ such that $\widetilde{\phi_{1}}(d(x, M)) \leq$ $\tilde{V}(x, r) \leq \tilde{\phi}_{2}(d(x, M))$ for all $(x, r) \in \tilde{X}_{1} \times R^{+}$.

2) For every $p\left(\cdot, a, r_{0}\right) \in S$ with $a \in \tilde{A}_{1}, \tilde{V}\left(p\left(r, a, r_{0}\right), r\right)$ is nonincreasing for all $r \geq r_{0}$.

From 1) and 2) above, we conclude that for any $r \in$ $\left[r_{n}, r_{n+1}\right)$, it is true that

$$
\begin{aligned}
& \tilde{\phi}_{1}\left(d\left(p\left(r, a, r_{0}\right), M\right)\right) \leq \tilde{V}\left(p\left(r, a, r_{0}\right), r\right) \\
& \leq \tilde{V}\left(p\left(r_{n}, a, r_{0}\right), r_{n}\right) \leq \tilde{\phi}_{2}\left(d\left(p\left(r_{n}, a, r_{0}\right), M\right)\right)
\end{aligned}
$$

which implies that

$$
\left.d\left(p\left(r, a, r_{0}\right), M\right)\right) \leq{\widetilde{\phi_{1}}}^{-1}\left[\tilde{\phi}_{2}\left(d\left(p\left(r_{n}, a, r_{0}\right), M\right)\right)\right]
$$

for all $r \in\left[r_{n}, r_{n+1}\right)$, and $n=0,1, \cdots$.

By the result in [16, Problem 3.8.9], there exists a function $\phi \in K$ defined on $\left[0, h_{0}\right]$, for some $h_{0}>0$, and another function $\sigma \in L$ such that

$$
d\left(p\left(r, a, r_{0}\right), M\right)<\phi(d(a, M)) \sigma\left(r-r_{0}\right)
$$

for all $p\left(\cdot, a, r_{0}\right) \in S$ and all $r \geq r_{0}$, where $d(a, M)<h_{0}$. Define $X_{1}=\left\{x \in \widetilde{A_{1}}: d(x, M)<h_{0}\right\}$, and

$$
A_{1}=\left\{\begin{array}{cl}
\left\{a \in X_{1}: d(a, M)<\phi^{-1}\left(h_{0}\right)\right\}, & \text { if } \phi\left(h_{0}\right)>h_{0} \\
X_{1}, & \text { otherwise. }
\end{array}\right.
$$

We are now ready to define the Lyapunov function $V\left(x, r_{0}\right)$ for $\left(x, r_{0}\right) \in X_{1} \times R^{+}$. Since for any $\left(x, r_{0}\right) \in X_{1} \times R^{+}$, there exists a unique motion $p\left(\cdot, x, r_{0}\right)$ which is continuous everywhere on $R^{+}$except on $E=\left\{r_{0}, r_{1}, \cdots,\right\}$, we define

$$
V\left(x, r_{0}\right)=\sum_{k=0}^{\infty} u\left(d\left(p\left(r_{k}, x, r_{0}\right), M\right)\right)
$$

where $u \in K$ will be specified later in such a manner that the above summation will converge. Obviously, $V\left(x, r_{0}\right) \geq$ $u\left(d\left(p\left(r_{0}, x, r_{0}\right), M\right)\right)=u(d(x, M))$. Hence, if we define $\phi_{1}=u$, then $V\left(x, r_{0}\right) \geq \phi_{1}(d(x, M))$ is true for all $\left(x, r_{0}\right) \in$ $X_{1} \times R^{+}$.

Consider $p\left(\cdot, x, r_{0}\right) \in S$ and the corresponding set $E=\left\{r_{1}, r_{2}, \cdots\right\}$. If we denote $\tilde{x}=p\left(r_{n}, a, r_{0}\right)$, and $\tilde{r_{0}}=r_{n}$ for some $n \geq 1$, we know there exists a unique motion $\tilde{p}\left(\cdot, \widetilde{x}, \tilde{r_{0}}\right) \in \bar{S}$ which is continuous everywhere on $r \geq \widetilde{r_{0}}$ except on $\left\{\tilde{r}_{1}, \tilde{r}_{2}, \cdots\right\}$. By the definition of $V$ given by (12), we know that $V\left(\tilde{x}, \tilde{r_{0}}\right)=\sum_{k=0}^{\infty} u\left(d\left(\tilde{p}\left(\tilde{r_{k}}, \tilde{x}, \tilde{r_{0}}\right), M\right)\right)$. However, by the uniqueness property, we know that $\tilde{r_{k}}=r_{n+k}$, and $\tilde{p}\left(\tilde{r_{k}}, \tilde{x}, \tilde{r_{0}}\right)=p\left(r_{n+k}, p\left(r_{n}, a, r_{0}\right), r_{n}\right)=$ $p\left(r_{n+k}, a, r_{0}\right)$. Therefore, it is clear that $V\left(p\left(r_{n}, a, r_{0}\right), r_{n}\right)=$ $\sum_{k=n}^{\infty} u\left(d\left(p\left(r_{k}, a, r_{0}\right), M\right)\right)$. It follows that

$D V\left(p\left(r_{n}, a, r_{0}\right), r_{n}\right)$

$=\left[V\left(p\left(r_{n+1}, a, r_{0}\right), r_{n+1}\right)-V\left(p\left(r_{n}, a, r_{0}\right), r_{n}\right)\right] /\left(r_{n+1}-r_{n}\right)$

$=\left[\sum_{k=n+1}^{\infty} u\left(d\left(p\left(r_{k}, a, r_{0}\right), M\right)\right)\right.$

$\left.-\sum_{k=n}^{\infty} u\left(d\left(p\left(r_{k}, a, r_{0}\right), M\right)\right)\right] /\left(r_{n+1}-r_{n}\right)$

$=-u\left(d\left(p\left(r_{n}, a, r_{0}\right), M\right)\right) /\left(r_{n+1}-r_{n}\right)$

for $n=0,1, \cdots$. Since $r_{n+1}-r_{n} \leq L$ by Assumption 4.2-2), it follows that

$$
\begin{aligned}
& D V\left(p\left(r_{n}, a, r_{0}\right), r_{n}\right) \leq-u\left(d\left(p\left(r_{n}, a, r_{0}\right), M\right)\right) / L \\
& =-\phi_{3}\left(d\left(p\left(r_{n}, a, r_{0}\right), M\right)\right)
\end{aligned}
$$

where we defined $\phi_{3}=u / L$.

We now show how to choose $u \in K$ so that the infinite summation (12) converges. It follows from (11) that for any $\left(x, r_{0}\right) \in X_{1} \times R^{+}$, we have

$$
\begin{aligned}
& u\left(d\left(p\left(r, x, r_{0}\right), M\right)\right)<u\left(\phi(d(x, M)) \sigma\left(r-r_{0}\right)\right) \\
& \leq[u(\phi(d(x, M)) \sigma(0))]^{\frac{1}{2}}\left[u\left(\phi\left(h_{0}\right) \sigma\left(r-r_{0}\right)\right)\right]^{\frac{1}{2}} .
\end{aligned}
$$

Let $\beta(\tau)=\phi\left(h_{0}\right) \sigma(\tau)$. Then $\beta \in L$. Hence, by Lemma 4.1, there exists an $\alpha \in K$ defined on $[0, \beta(0)]$ such that $\sum_{i=0}^{\infty} \alpha\left(\beta\left(r_{i+1}-r_{i}\right)\right)<\infty$. If we define $u(r)=[\alpha(r)]^{2}$, then it follows that

$$
\begin{aligned}
& {\left[u\left(\phi\left(h_{0}\right) \sigma\left(r-r_{0}\right)\right)\right]^{\frac{1}{2}}} \\
& =\left[\alpha\left(\phi\left(h_{0}\right) \sigma\left(r-r_{0}\right)\right)\right]^{\frac{1}{2}}=\alpha\left(\beta\left(r-r_{0}\right)\right) .
\end{aligned}
$$

Hence, we conclude from (12)-(14), and (9) that $V\left(x, r_{0}\right)=$ $\sum_{i=0}^{\infty} u\left(d\left(p\left(r_{i}, x, r_{0}\right), M\right)\right)<\sum_{i=0}^{\infty} \quad[u(\phi(d(x, M)) \times$ $\sigma(0))]^{\frac{1}{2}} \times\left[u\left(\phi\left(h_{0}\right) \times \sigma\left(r_{i}-r_{0}\right)\right)\right]^{\frac{1}{2}}=[u(\phi(d(x, M)) \times$ $\sigma(0))]^{\frac{1}{2}} \times \sum_{i=0}^{\infty} \alpha\left(\beta\left(r_{i}-r_{0}\right)\right)<[u(\phi(d(x, M)) \times \sigma(0))]^{\frac{1}{2}} \times$ $[1+1 /(1-\exp (-l))]$. 
If we define $\phi_{2} \in K$ by $\phi_{2}(r)=[u(\phi(\sigma(0) r))]^{\frac{1}{2}}[1+1 /(1-$ $\exp (-l))]$ then it follows that $V\left(x, r_{0}\right) \leq \phi_{2}(d(x, M))$. Thus we have proved parts 1) and 2) of Theorem 4.6.

To prove part 3) of the theorem, let $r \in\left[r_{n}, r_{n+1}\right)$. We have already shown that $V\left(p\left(r, a, r_{0}\right), r\right) \leq \phi_{2}\left(d\left(p\left(r, a, r_{0}\right), M\right)\right)$. Furthermore, since $a \in A_{1} \subset \tilde{A}_{1}$, (10) is satisfied. Hence, we know that

$$
V\left(p\left(r, a, r_{0}\right), r\right) \leq \phi_{2} \circ \tilde{\phi}_{1}^{-1} \circ \tilde{\phi}_{2}\left(d\left(p\left(r_{n}, a, r_{0}\right), M\right)\right) .
$$

On the other hand, we have also shown that $V\left(p\left(r_{n}\right.\right.$, $\left.\left.a, r_{0}\right), r_{n}\right) \geq \phi_{1}\left(d\left(p\left(r_{n}, a, r_{0}\right), M\right)\right)$, which implies that

$$
\phi_{1}^{-1} \circ V\left(p\left(r_{n}, a, r_{0}\right), r_{n}\right) \geq d\left(p\left(r_{n}, a, r_{0}\right), M\right) .
$$

Combining (15) and (16), we obtain that $V\left(p\left(r, a, r_{0}\right), r\right) \leq$ $\phi_{2} \circ \tilde{\phi}_{1}^{-1} \circ \tilde{\phi}_{2} \circ \phi_{1}^{-1}\left(V\left(p\left(r_{n}, a, r_{0}\right), r_{n}\right)\right)$ for all $r \in\left[r_{n}, r_{n+1}\right)$, $n=0,1, \cdots$, and all $\left(a, r_{0}\right) \in A_{1} \times R^{+}$. If we now define $f=\phi_{2} \circ \tilde{\phi}_{1}^{-1} \circ \tilde{\phi}_{2} \circ \phi_{1}^{-1}$, then $f \in C\left(R^{+}, R^{+}\right), f(0)=0$, and $V\left(p\left(r, a, r_{0}\right), r\right) \leq f\left(V\left(p\left(r_{n}, a, r_{0}\right), r_{n}\right)\right)$. This concludes the proof of the theorem.

Although converse theorems are in general not very useful in constructing Lyapunov functions, their importance in stability analysis cannot be overemphasized. In particular, such results ensure the existence of Lyapunov functions with appropriate properties under suitable conditions. Furthermore, converse theorems tell us that under a given set of hypotheses, a stability result is as good as you can possibly expect. For additional converse theorems, refer to [22]-[28] and [30].

Before proceeding to applications, we wish to point to the generality of all results presented above. These allow analysis of finite-dimensional as well as infinite-dimensional systems.

\section{Application to Nonlinear Sampled-Data Systems}

Our primary objective in this section is to present a detailed application of the stability theory developed herein to the most widely known class of HDS's, sampled-data systems. The qualitative analysis of sampled-data control systems has been of great interest in the past, and because of significant advances in digital controller technology it continues to be of current interest (see, e.g., [10]-[12]). These investigations are primarily concerned with linear models. In the present section we apply the results of Section IV in the stability analysis of sampled-data control systems of the type considered in Example 1 (refer to Section III), given by equations of the form

$$
\left\{\begin{array}{l}
\dot{x}(r)=f(x(r))+B u(k), r \in[k, k+1) \\
u(k+1)=C u(k)+D x(k), k=0,1,2, \cdots
\end{array}\right.
$$

where all symbols in (17) are as defined in (1). We note that since $f(0)=0,\left(x^{T}, u^{T}\right)^{T}=\left(0^{T}, 0^{T}\right)^{T}$ is an equilibrium of (17).

In this section we will show that the asymptotic stability of the equilibrium $\left(x^{T}, u^{T}\right)^{T}=\left(0^{T}, 0^{T}\right)^{T}$ of (17) can be deduced from the asymptotic stability of the trivial solution of the associated linear system given by

$$
\left\{\begin{array}{l}
\dot{x}(r)=A x(r)+B u(k), r \in[k, k+1) \\
u(k+1)=C u(k)+D x(k), k=0,1,2, \cdots
\end{array}\right.
$$

where $A \in R^{n \times n}$ denotes the Jacobian of $f$ evaluated at $x=0$, i.e.,

$$
A=\left[\frac{\partial f}{\partial x}(0)\right]_{n \times n} .
$$

We note that in (17) the components of the state, $x, u$, are directly accessible as subsystem outputs (of the plant and the digital controller, respectively). When this is not the case, transducers are used to measure the states indirectly, resulting in linear output equations, as given for example in the system description

$$
\left\{\begin{array}{l}
\dot{x}(r)=f(x(r))+B w(k), r \in[k, k+1) \\
y(r)=M x(r), r \in[k, k+1) \\
u(k+1)=C u(k)+D y(k), k=0,1, \cdots \\
w(k)=P u(k), k=0,1, \cdots
\end{array}\right.
$$

where $y \in R^{l}, w \in R^{s}$, and $M$ and $P$ are real matrices of appropriate dimensions. By using the methodology employed herein, it is possible to establish a stability result of the type described above for (17), using the linearization of (20) about the equilibrium $\left(x^{T}, u^{T}\right)^{T}=\left(0^{T}, 0^{T}\right)^{T}$. We will not pursue this.

For the linear sampled-data system (18), we have the following result.

Lemma 5.1: The equilibrium $\left(x^{T}, u^{T}\right)^{T}=\left(0^{T}, 0^{T}\right)^{T}$ of the linear HDS determined by the system of equations (18) is uniformly asymptotically stable if and only if the matrix

$$
H \triangleq\left[\begin{array}{cc}
H_{1} & H_{2} \\
D & C
\end{array}\right]
$$

is Schur stable, where

$$
H_{1}=e^{A}, \quad H_{2}=\int_{0}^{1} e^{A(1-\tau)} d \tau B .
$$

The conclusion of Lemma 5.1 is well known (refer, e.g., to [10] and [11]).

We are now in a position to prove the main result of the present section.

Theorem 5.1: The equilibrium $\left(x^{T}, u^{T}\right)^{T}=\left(0^{T}, 0^{T}\right)^{T}$ of (17) is uniformly asymptotically stable if the equilibrium $\left(x^{T}, u^{T}\right)^{T}=\left(0^{T}, 0^{T}\right)^{T}$ of the linear dynamical system determined by the system of equations given in (18) is uniformly asymptotically stable, or equivalently, if the matrix $H$ given in (21) is Schur stable.

Proof: Since $f \in C^{1}\left[R^{n}, R^{n}\right]$ and $f(0)=0$, we can represent $f$ as

$$
f(x)=A x+g(x)
$$

where the matrix $A$ is given in (19) and $g \in C\left[R^{n}, R^{n}\right]$ satisfies

$$
\lim _{\|x\| \rightarrow 0} \frac{\|g(x)\|}{\|x\|}=0 .
$$

It follows from (24) that there exits a $\delta>0$ such that

$$
\|g(x)\| \leq\|x\|
$$


whenever $\|x\| \leq \delta$. If we let $\delta_{1}=\frac{e^{-(\|A\|+1)}}{\|B\|+1} \delta$, then we can conclude that for any $k \in N$, it is true that $\|x(r)\| \leq \delta$ for all $r \in[k, k+1]$ whenever $\|x(k)\| \leq \delta_{1}$ and $\|u(k)\| \leq \delta_{1}$. For otherwise, there must exist an $r_{0} \in(k, k+1)$ such that $\left\|x\left(r_{0}\right)\right\|=\delta$ and $\|x(r)\| \leq \delta$ for all $r \in\left[k, r_{0}\right]$. We will prove that this is impossible. Since for any $r \in[k, k+1]$ we have that

$$
x(r)=x(k)+\int_{k}^{r}[A x(\tau)+g(x(\tau))+B u(k)] d \tau
$$

it is true that $\|x(r)\| \leq\|x(k)\|+\int_{k}^{r}[\|A\| \cdot\|x(\tau)\|+\|g(x(\tau))\|+$ $\|B\| \cdot\|u(k)\|] d \tau$ for all $r \in[k, k+1]$. In particular, when $r \in\left[k, r_{0}\right]$, we have that

$$
\begin{aligned}
& \|x(r)\| \leq(\|x(k)\|+(r-k)\|B\| \cdot\|u(k)\|) \\
& +\int_{k}^{r}[\|A\| \cdot\|x(\tau)\|+\| g(x(\tau) \|] d \tau \\
& \leq(\|x(k)\|+\|B\| \cdot\|u(k)\|) \\
& +\int_{k}^{r}[\|A\| \cdot\|x(\tau)\|+\quad\|x(\tau)\|] d \tau
\end{aligned}
$$

where we have used in the last step of (27) the fact that $\|g(x(\tau))\| \leq\|x(\tau)\|$, since $\|x(\tau)\| \leq \delta$ for all $\tau \in\left[k, r_{0}\right]$, by assumption. By Gronwall's inequality (see, e.g., [20]), (27) implies that

$$
\|x(r)\| \leq(\|x(k)\|+\|B\| \cdot\|u(k)\|) e^{(\|A\|+1)(r-k)}
$$

for all $r \in\left[k, r_{0}\right]$. Hence

$$
\begin{aligned}
& \left\|x\left(r_{0}\right)\right\| \leq(\|x(k)\|+\|B\| \cdot\|u(k)\|) e^{(\|A\|+1)\left(r_{0}-k\right)} \\
& \leq(1+\|B\|) \delta_{1} e^{(\|A\|+1)\left(r_{0}-k\right)} \\
& =(1+\|B\|) \frac{e^{-(\|A\|+1)}}{\|B\|+1} \delta \cdot e^{(\|A\|+1)\left(r_{0}-k\right)} \\
& =\delta \cdot e^{(\|A\|+1)\left(r_{0}-k-1\right)}<\delta
\end{aligned}
$$

since $r_{0}<k+1$. Inequality (29) contradicts the assumption that $\left\|x\left(r_{0}\right)\right\|=\delta$. Therefore, we have shown that for any $k \in N$, it is true that $\|x(r)\| \leq \delta$ for all $r \in[k, k+1]$ whenever $\|x(k)\| \leq \delta_{1}$ and $\|u(k)\| \leq \delta_{1}$. In view of (25), we can further conclude that

$$
\|g(x(r))\| \leq\|x(r)\|
$$

for all $r \in[k, k+1]$ whenever $\|x(k)\| \leq \delta_{1}$ and $\|u(k)\| \leq \delta_{1}$. Equation (30) implies that (27) and (28) hold for all $r \in$ $[k, k+1]$, assuming that $\|x(k)\| \leq \delta_{1}$ and $\|u(k)\| \leq \delta_{1}$. Therefore, it follows from (28) that

$$
\|x(r)\| \leq(\|x(k)\|+\|u(k)\|) \cdot(1+\|B\|) e^{(\|A\|+1)}
$$

for all $r \in[k, k+1]$, assuming that $\|x(k)\| \leq \delta_{1}$ and $\|u(k)\| \leq \delta_{1}$.

Since $f(x)=A x+g(x)$, the first equation in (17) can be written as

$$
\dot{x}(r)=A x(r)+g(x(r))+B u(k)
$$

for $r \in[k, k+1]$. The solution of equation (32) must have the form

$$
\begin{aligned}
x(r) & =e^{A(r-k)} x(k)+\int_{k}^{r} e^{A(r-\tau)} B u(k) d \tau \\
& +\int_{k}^{r} e^{A(r-\tau)} g(x(\tau)) d \tau
\end{aligned}
$$

for all $r \in[k, k+1]$. Specifically, when $r=k+1$, we have that

$$
\begin{aligned}
x(k+1)= & e^{A} x(k)+\int_{k}^{k+1} e^{A(k+1-\tau)} B u(k) d \tau \\
& +\int_{k}^{k+1} e^{A(k+1-\tau)} g(x(\tau)) d \tau \\
= & e^{A} x(k)+\int_{0}^{1} e^{A(1-\tau)} d \tau \cdot B u(k) d \tau+\Delta(k)
\end{aligned}
$$

where

$$
\Delta(k)=\int_{k}^{k+1} e^{A(k+1-\tau)} g(x(\tau)) d \tau .
$$

Before proceeding further, we require the following intermediate result.

Claim 1: For any given $\epsilon>0$, there exists a $\delta_{3}>0$, $\delta_{3} \leq \delta_{1}$, such that for any $k \in N$ it is true that $\|\Delta(k)\|<$ $\epsilon(\|x(k)\|+\|u(k)\|)$ whenever $\|x(k)\|<\delta_{3}$ and $\|u(k)\|<\delta_{3}$.

Proof: For the given $\epsilon>0$, we choose $\epsilon_{1}>0$ such that $\epsilon=\epsilon_{1} \cdot e^{(2\|A\|+1)}(1+\|B\|)$. We know by (24) that there must exist a $\delta_{2}>0$ such that

$$
\|g(x)\|<\epsilon_{1}\|x\|
$$

whenever $\|x\|<\delta_{2}$. We choose

$$
\delta_{3}=\min \left\{\delta_{1}, \frac{\delta_{2}}{2(1+\|B\|) e^{(\|A\|+1)}}\right\} .
$$

Then, whenever $\|x(k)\| \leq \delta_{3}$ and $\|u(k)\| \leq \delta_{3}$, it is true by (28) that

$$
\begin{aligned}
& \|x(r)\| \leq(\|x(k)\|+\|u(k)\|) \cdot(1+\|B\|) e^{(\|A\|+1)} \\
& \leq 2 \delta_{3}(1+\|B\|) e^{(\|A\|+1)} \leq \delta_{2}
\end{aligned}
$$

for all $r \in[k, k+1]$. Combining (37), (36), and (31), we obtain that

$$
\begin{aligned}
& \|g(x(r))\| \leq \epsilon_{1}\|x(r)\| \\
& \leq \epsilon_{1}(\|x(k)\|+\|u(k)\|) \cdot(1+\|B\|) e^{(\|A\|+1)}
\end{aligned}
$$

for all $r \in[k, k+1]$ whenever $\|x(k)\| \leq \delta_{3}$ and $\|u(k)\| \leq \delta_{3}$. Hence, for $\Delta(k)$ given by (35), we know that

$$
\begin{aligned}
& \|\Delta(k)\| \leq \int_{k}^{k+1} e^{\|A\|} \cdot\|g(x(\tau))\| d \tau \\
& \leq e^{\|A\|} \cdot \epsilon_{1}(\|x(k)\|+\|u(k)\|) \cdot(1+\|B\|) e^{(\|A\|+1)} \\
& =\epsilon \cdot(\|x(k)\|+\|u(k)\|)
\end{aligned}
$$

whenever the conditions $\|x(k)\| \leq \delta_{3}$ and $\|u(k)\| \leq \delta_{3}$ are satisfied, concluding the proof of Claim 1 .

We are now in a position to apply the results of Sections III and IV to prove the present theorem. As discussed in Example 1 of Section III-B, (17) [or, equivalently, (1)] can be regarded as a HDS defined on the time space $T=\left\{(r, k) \in R^{2}: r \geq\right.$ $0, k=[r]\}$. The state for this hybrid system, denoted by $p(t)$, is given by $p(t)=\left[x(r)^{T}, u(k)^{T}\right]^{T}$ where $t=(r, k) \in T$. This hybrid system can be embedded into a dynamical system defined on $R^{+}$by the embedding mapping $g_{0}: T \rightarrow R^{+}$such that $g_{0}(t)=r$ for any $t=(r, k) \in T$ (as was explained in 
Section III-B). If we denote the state in the new embedded dynamical system defined on $R^{+}$by $w \in R^{n+m}$, then

$$
w(r)=\left[x(r)^{T}, u(k)^{T}\right]^{T}
$$

where $k=[r]$. We will show that $w=0$ is an asymptotically stable equilibrium of this dynamical system. Therefore, $\left(x^{T}, u^{T}\right)^{T}=\left(0^{T}, 0^{T}\right)^{T}$ is a uniformly asymptotically stable equilibrium of the original hybrid system (17).

Since by assumption $H$ is Schur stable, where $H$ is given in (21), we know that there exists a positive definite matrix $P$ such that $H^{T} P H-P=-2 I$, where $I \in R^{(n+m) \times(n+m)}$ denotes the identity matrix. We now define the Lyapunov function

$$
V(w, r)=w^{T} P w
$$

and we show that $V(w(r), r)$ satisfies all the conditions of Theorem 4.1 for any motion $w(r)$. Clearly, $w(r)$ is a motion which is continuous everywhere on $R^{+}$except on $N$, the set of nonnegative integers. For any $k \in N$, it is known by (34) and (17) that

$$
w(k+1)=H w(k)+m(k)
$$

where $H$ is given by $(21), m(k)$ is given by

$$
m(k) \triangleq\left(\Delta(k)^{T}, 0^{T}\right)^{T}
$$

and $\Delta(k)$ is given by (35). It now follows that

$$
\begin{aligned}
& V(w(k+1), k+1)-V(w(k), k) \\
&= w^{T}(k+1) P w(k+1)-w^{T}(k) P w(k) \\
&= {[H w(k)+m(k)]^{T} P[H w(k)+m(k)]-w^{T}(k) P w(k) } \\
&= w^{T}(k)\left[H^{T} P H-P\right] w(k)+2 m^{T}(k) P H w(k) \\
&+m^{T}(k) P m(k) \\
&=-2\|w(k)\|^{2}+2 m^{T}(k) P H w(k)+m^{T}(k) P m(k) \\
& \leq-2\|w(k)\|^{2}+2\|m(k)\| \cdot\|P H\| \cdot\|w(k)\| \\
&+\|m(k)\|^{2} \cdot\|P\| .
\end{aligned}
$$

By the definition of $m(k)$, we know that $\|m(k)\|=\|\Delta(k)\|$. Furthermore, by Claim 1, if we choose an $\epsilon_{0}>0$ such that

$$
2 \sqrt{2} \epsilon_{0}\|P H\|+2 \epsilon_{0}^{2}\|P\|<2
$$

then there exists a $\delta_{3}\left(\epsilon_{0}\right)$ such that $\|\Delta(k)\|<\epsilon_{0}(\|x(k)\|+$ $\|u(k)\|)$ whenever $\|x(k)\|<\delta_{3}\left(\epsilon_{0}\right)$ and $\|u(k)\|<\delta_{3}\left(\epsilon_{0}\right)$. Therefore, whenever $\|w(k)\|<\delta_{3}\left(\epsilon_{0}\right)$ (noticing that $\left.\|w(k)\|^{2}=\|u(k)\|^{2}+\|x(k)\|^{2}\right)$, it is true that

$$
\begin{aligned}
\|m(k)\| & =\|\Delta(k)\|<\epsilon_{0}(\|x(k)\|+\|u(k)\|) \\
& \leq \epsilon_{0} \sqrt{2}\|w(k)\|
\end{aligned}
$$

must hold. Combining (44) and (46), we conclude that

$$
\begin{aligned}
& V(w(k+1), k+1)-V(w(k), k) \\
&<-2\|w(k)\|^{2}+2 \sqrt{2} \epsilon_{0}\|P H\| \cdot\|w(k)\|^{2} \\
&+2 \epsilon_{0}^{2}\|P\| \cdot\|w(k)\|^{2} \\
&=\|w(k)\|^{2}\left(-2+2 \sqrt{2} \epsilon_{0}\|P H\|+2 \epsilon_{0}^{2} \cdot\|P\|\right)
\end{aligned}
$$

whenever $\|w(k)\|<\delta_{3}\left(\epsilon_{0}\right)$. Before concluding the proof, we require another intermediate result.
Claim 2: For any $k_{0} \in N$, (47) holds for all $k \geq k_{0}$ whenever

$$
\left\|w\left(k_{0}\right)\right\|<\left(\frac{\lambda_{\min }(P)}{\lambda_{\max }(P)}\right)^{\frac{1}{2}} \delta_{3}\left(\epsilon_{0}\right)
$$

where $\lambda_{\min }(P)$ and $\lambda_{\max }(P)$ denote the minimum and maximum eigenvalues of $P$, respectively.

Proof: Equation (48) implies that $V\left(w\left(k_{0}\right), k_{0}\right) \leq$ $\lambda_{\max }(P)\left\|w\left(k_{0}\right)\right\|^{2}<\lambda_{\min }(P) \delta_{3}\left(\epsilon_{0}\right)$. Since $\left\|w\left(k_{0}\right)\right\|<$ $\delta_{3}\left(\epsilon_{0}\right)$ is satisfied, we know by (47) that $V\left(w\left(k_{0}+1\right), k_{0}+1\right)$ is less than $V\left(w\left(k_{0}\right), k_{0}\right)$ because of (45). Therefore

$$
V\left(w\left(k_{0}+1\right), k_{0}+1\right)<\lambda_{\min }(P) \delta_{3}\left(\epsilon_{0}\right)
$$

must be satisfied as well. Furthermore, since (49) implies that $\left\|w\left(k_{0}+1\right)\right\|<\delta_{3}\left(\epsilon_{0}\right)$, it follows that $V\left(w\left(k_{0}+2\right), k_{0}+2\right)$ is less than $V\left(w\left(k_{0}+1\right), k_{0}+1\right)$, and $\left\|w\left(k_{0}+2\right)\right\|$ is less than $\delta_{3}\left(\epsilon_{0}\right)$. By induction, it follows that $\|w(k)\|<\delta_{3}\left(\epsilon_{0}\right)$ for all $k \geq k_{0}$. Hence (47) is satisfied for all $k \geq k_{0}$ as long as (48) is true. This concludes the proof of Claim 2.

By Claim 2 we know that for any motion $w(r)$, condition 2) of Theorem 4.1 is satisfied for $r \geq k_{0}$, as long as (48) is true. Furthermore, it can be shown that (31) implies that

$V(w(r), r)$

$$
\leq \frac{\lambda_{\max }(P)}{\lambda_{\min }(P)}\left[1+(1+\|B\|) e^{(\|A\|+1)} \sqrt{2}\right]^{2} V(w(k), k)
$$

for all $r \geq k_{0}$, and $k=[r]$, by noticing that $\|w(k)\|<$ $\delta_{3}\left(\epsilon_{0}\right)<\delta_{1}$ whenever (48) is satisfied. Hence, if we define $f \in C\left(R^{+}, R^{+}\right)$as

$$
f(\rho)=\frac{\lambda_{\max }(P)}{\lambda_{\min }(P)}\left[1+(1+\|B\|) e^{(\|A\|+1)} \sqrt{2}\right]^{2} \rho
$$

then condition 1) of Theorem 4.1 will also be satisfied whenever the initial condition for (47) holds. Noting that $\delta_{3}\left(\epsilon_{0}\right)$ is independent of $k_{0}$, it follows from Theorem 4.1, that the equilibrium $\left(x^{T}, u^{T}\right)^{T}=\left(0^{T}, 0^{T}\right)^{T}$ of (17) is uniformly asymptotically stable if the matrix $H$ [given by (21)] is Schur stable. This concludes the proof of the theorem.

For further results which are in the spirit of Theorem 5.1, refer to [26]. Other sources that address the present problem in a different context, using methods that differ significantly from the present approach, include, e.g., [32].

\section{Systems WITH IMPULSE EFFECTS}

There are numerous examples of evolutionary systems which at certain instants of time are subjected to rapid changes. In the simulations of such processes it is frequently convenient and valid to neglect the durations of the rapid changes and to assume that the changes can be represented by state jumps. Examples of such systems arise in mechanics (e.g., the behavior of a buffer subjected to a shock effect, the behavior of clock mechanisms, the change of velocity of a rocket at the time of separation of a stage, and so forth), in radio engineering and communication systems (where the generation of impulses of various forms is common), in biological systems (where, e.g., sudden population changes due to external effects occur frequently), in control theory (e.g., 
impulse control, robotics, etc.), and the like. For additional specific examples, refer, e.g., to [21] and [31].

Appropriate mathematical models for processes of the type described above are so-called systems with impulse effects. The qualitative behavior of such systems has been investigated extensively in the literature (refer to [21] and the references cited in [21]). In the present section we will establish qualitative results for systems with impulse effects which in general are less conservative than existing results [21], [31].

We will concern ourselves with finite-dimensional systems described by ordinary differential equations with impulse effects. For this reason we will let $X=R^{n}$ in the present section, the metric $d$ will be assumed to be determined by norm $\|\cdot\|$, and $T=R^{+}$.

The class of systems with impulse effects under investigation can be described by equations of the form

$$
\left\{\begin{array}{l}
\frac{d x}{d t}=f(x, t), t \neq \tau_{k} \\
\Delta x=I_{k}(x), t=\tau_{k}
\end{array}\right.
$$

where $x \in X=R^{n}$ denotes the state, $f \in C\left[R^{n} \times\right.$ $\left.R^{+}, R^{n}\right]$ satisfies a Lipschitz condition with respect to $x$ which guarantees the existence and uniqueness of solutions of (50) for given initial conditions, $E=\left\{\tau_{1}, \tau_{2}, \cdots: \tau_{1}<\right.$ $\left.\tau_{2}<\cdots\right\} \subset R^{+}$is an unbounded closed discrete subset of $R^{+}$which denotes the set of times when jumps occur, and $I_{k} \in C\left[R^{n}, R^{n}\right]$ denotes the incremental change of the state at the time $\tau_{k}$. It should be pointed out that in general $E$ depends on a specific motion and that for different motions, the corresponding sets $E=\left\{\tau_{1}, \tau_{2}, \cdots: \tau_{1}<\tau_{2}<\cdots\right\} \subset R^{+}$ are in general different. The function $\phi:\left[t_{0}, \infty\right) \rightarrow R^{n}$ is said to be a solution of the system with impulse effects (50) if 1) $\phi(t)$ is left continuous on $\left[t_{0}, \infty\right)$ for some $t_{0} \geq 0$; 2) $\phi(t)$ is differentiable and $\frac{d \phi}{d t}(t)=f(\phi(t), t)$ everywhere on $\left(t_{0}, \infty\right)$ except on an unbounded, closed, discrete subset $E=\left\{\tau_{1}, \tau_{2}, \cdots: \tau_{1}<\tau_{2}<\cdots\right\} \subset R^{+}$; and 3) for any $t=\tau_{k} \in E, \phi\left(t^{+}\right)=\phi(t)+I_{k}(\phi(t))$, where $\phi\left(t^{+}\right)$denotes the right limit of $\phi$ at $t$, i.e., $\phi\left(t^{+}\right)=\lim _{r \rightarrow t^{+}} \phi(r)$.

If for (50), we assume further that $f(0, t)=0$ for all $t \in R^{+}$, and $I_{k}(0)=0$ for all $k \in N$, then it is clear that $x=0$ is an equilibrium. For this equilibrium, the following results have been established in [21, Th. 13.1 and 13.2].

Proposition 6.1: Assume that for (50) satisfying $f(0, t)=$ 0 and $I_{k}(0)=0$ for all $t \in R^{+}$and $k \in N$, there exists a $V: X \times R^{+} \rightarrow R^{+}$and $\phi_{1}, \phi_{2} \in K$ such that $\phi_{1}(\|x\|) \leq V(x, t) \leq \phi_{2}(\|x\|)$ for all $(x, t) \in X \times R^{+}$.

$1)$ : If for any solution $x(t)$ of (50), which is defined on $\left[t_{0}, \infty\right)$, it is true that $V(x(t), t)$ is left continuous on $\left[t_{0}, \infty\right)$ and is differentiable everywhere on $\left(t_{0}, \infty\right)$ except on an unbounded closed discrete set $E=\left\{\tau_{1}, \tau_{2}, \cdots\right\}$, where $E$ is the set of the times when jumps occur for $x(t)$, and if it is also true that

$$
\left\{\begin{array}{l}
\frac{d V(x(t), t)}{d t} \leq 0 \text { for } t \neq \tau_{k}, \text { and } \\
V\left(x\left(\tau_{k}\right)+I_{k}\left(x\left(\tau_{k}\right)\right), \tau_{k}^{+}\right) \leq V\left(x\left(\tau_{k}\right), \tau_{k}\right)
\end{array}\right.
$$

for all $t_{k} \in E$, then the equilibrium $x=0$ of (50) is uniformly stable.
2): If in addition, we assume that there exists a $\phi_{3} \in K$ such that

$$
\frac{d V(x(t), t)}{d t} \leq-\phi_{3}(\|x(t)\|), t \neq \tau_{k}
$$

then the equilibrium $x=0$ of (50) is uniformly asymptotically stable.

The above proposition provides a sufficient condition for the uniform stability and the uniform asymptotic stability of the equilibrium $x=0$ of (50). It is shown in [21] that under additional conditions, the above results also constitute necessary conditions (see [21, Ch. 15]). One critical assumption in these necessary conditions is that the impulse effects occur at fixed instants of time, i.e., in (50) the set $E=\left\{\tau_{1}, \tau_{2}, \cdots\right\}$ is independent of the different solutions. This assumption may be unrealistic, since in applications it is often the case that the impulse effects occur when a given motion reaches some threshold conditions. Accordingly, for different initial conditions, the sets of time instants when jumps in the motions will occur will, in general, vary.

It is easily shown that (50) is a special case of the HDS defined in Section III-A. Applying Theorem 4.1 to (50), we obtain the following result.

Theorem 6.1: Assume that for $(50) f(0, t)=0$ and $I_{k}(0)=$ 0 for all $t \in R^{+}$and $k \in N$, that there exists an $h \in$ $C\left[R^{+}, R^{+}\right]$such that $h(0)=0$ and a $V: X \times R^{+} \rightarrow R^{+}$ and $\phi_{1}, \phi_{2} \in K$ such that $\phi_{1}(\|x\|) \leq V(x, t) \leq \phi_{2}(\|x\|)$ for all $(x, t) \in X \times R^{+}$.

1): Assume that for any solution $x(t)$ of (50) which is defined on $\left[t_{0}, \infty\right), V(x(t), t)$ is left continuous on $\left[t_{0}, \infty\right)$ and is differentiable everywhere on $\left(t_{0}, \infty\right)$ except on an unbounded closed discrete set $E=\left\{\tau_{1}, \tau_{2}, \cdots\right\}$, where $E$ is the set of times when jumps occur for $x(t)$ and that $V\left(x\left(\tau_{n}\right)+\right.$ $\left.I_{n}\left(x\left(\tau_{n}\right)\right), \tau_{n}^{+}\right)$) (which is actually $\lim _{t \rightarrow \tau_{n}^{+}} V(x(t), t)$ ) is nonincreasing for $n=0,1, \cdots$ where

$$
\tau_{0} \triangleq t_{0}
$$

Furthermore, assume that $V(x(t), t) \leq h\left(V\left(x\left(\tau_{n}\right)+\right.\right.$ $\left.\left.I_{n}\left(x\left(\tau_{n}\right)\right), \tau_{n}^{+}\right)\right)$is true for all $t \in\left(\tau_{n}, \tau_{n+1}\right], n=0,1, \cdots$. Then the equilibrium $x=0$ of (50) is uniformly stable.

2): If in addition to 1), we assume that there exists a $\phi_{3} \in$ $K$ such that, $D V\left(x\left(\tau_{n}\right)+I_{n}\left(x\left(\tau_{n}\right)\right), \tau_{n}^{+}\right) \leq-\phi_{3}\left(\left\|x\left(\tau_{n}\right)\right\|\right)$ is true for all $n=0,1, \cdots$, where

$$
\begin{gathered}
D V\left(x\left(\tau_{n}\right)+I_{n}\left(x\left(\tau_{n}\right)\right), \tau_{n}^{+}\right) \triangleq \frac{1}{\tau_{n+1}-\tau_{n}}\left[V \left(x\left(\tau_{n+1}\right)\right.\right. \\
\left.\left.+I_{n+1}\left(x\left(\tau_{n+1}\right)\right), \tau_{n+1}^{+}\right)-V\left(x\left(\tau_{n}\right), \tau_{n}^{+}\right)\right]
\end{gathered}
$$

then the equilibrium $x=0$ of (50) is uniformly asymptotically stable.

In the interests of brevity, we omit the details of the proof of Theorem 6.1. For details concerning this proof and additional results on impulse systems, refer to [25].

Remarks 1): Theorem 6.1 is less conservative than Proposition 6.1. Specifically, in Proposition 6.1 the Lyapunov function $V$ is required to be monotonically nonincreasing everywhere except at the instants $\tau_{k}$ where impulses occur, and at every such $\tau_{k}$ the function $V$ is only allowed to decrease (jump downwards). On the other hand, in Theorem 6.1 we only 
require that the right limits of $V$ at times $\tau_{k}$, when jumps occur, be nonincreasing and that at all other times between $\tau_{k}$ and $\tau_{k+1}$ the Lyapunov function $V$ be bounded by the combination of a prespecified bounded function and the right limit of $V$ at $\tau_{k}$.

2): As pointed out earlier, a converse result for Proposition 6.1 was established in [21, Ch. 15] under the rather strong assumption that the impulse effects occur at fixed instances of times. For Theorem 6.1, however, we can establish a converse theorem, which involves considerably milder hypotheses (which are very similar to Assumptions 4.1 and 4.2.), by applying Theorem 4.6 (refer to [25]).

To demonstrate a specific application of Theorem 6.1, we consider the special case of (50) described by equations of the form

$$
\begin{cases}\frac{d x}{d t}=f(x), & t \neq \tau_{k} \\ \Delta x=B_{k} x, & t=\tau_{k}, k \in N\end{cases}
$$

where $f \in C^{1}\left[R^{n}, R^{n}\right]$, where it is assumed that $f(0)=0$, where $B_{k} \in R^{n \times n}$, and $\left\{\tau_{1}<\tau_{2}<\cdots\right\}$ denotes the discrete closed unbounded set of fixed instances (independent of specific trajectories) when impulse effects occur. A special class of (53) are systems described by

$$
\left\{\begin{aligned}
\frac{d x}{d t} & =A x, & & t \neq \tau_{k} \\
\Delta x & =B_{k} x, & & t=\tau_{k}, k \in N
\end{aligned}\right.
$$

where $B_{k}$ and $\tau_{k}$ are the same as in system (53) and $A \in R^{n \times n}$ is a constant matrix. Such systems have been investigated in [21, Ch. 4.2]. In particular, the following result was established in [21, Th. 4.3].

Proposition 6.2: The equilibrium $x=0$ of (54) is asymptotically stable if the condition 1) $0<l=\inf \left\{\tau_{k+1}-\tau_{k}\right\} \leq$ $\sup \left\{\tau_{k+1}-\tau_{k}\right\}=\lambda<\infty$ is satisfied, together with either the condition 2) $\left\|U_{j}\right\| \leq q<1(j \in N)$, or the condition 3) $\lim _{j \rightarrow \infty} U_{j}=U$, where it is assumed that the modulus of each eigenvalue of $U$ is smaller than one and where $U_{j} \triangleq\left(I+B_{j}\right) e^{A\left(\tau_{j}-\tau_{j-1}\right)}, j \in N$.

By applying Theorem 6.1 to (53), we obtain the following result.

Theorem 6.2: For (53), let $A$ denote the Jacobian of $f$ at $x=0$ [i.e., $A=\frac{\partial f}{\partial x}(0)$ ] and assume that the condition 1) and either condition 2) or condition 3) of Proposition 6.2 are satisfied for (54). Then, the equilibrium $x=0$ of (53) is asymptotically stable.

Remarks 1): Theorem 6.2 implies that when the linearization of (53) satisfies the sufficient conditions in Proposition 6.2 , which assure the asymptotic stability of the linear system (54) with impulse effects, then the equilibrium of the original nonlinear system (53) is also asymptotically stable.

2): The proof of Theorem 6.2 (which we omit due to space limitations) can be accomplished by using similar arguments as in the proof of Theorem 5.1; refer to [25] for the details of the proof of Theorem 6.2 .

\section{CONCluding REMARKS}

We have initiated a systematic study of the qualitative properties of HDS's. To accomplish this, we first formulated a general model for such systems which is suitable for qualitative investigations. Next, we defined in a natural manner various stability concepts of invariant sets and boundedness of motions for such systems. We then established sufficient conditions for uniform stability, uniform asymptotic stability, exponential stability, and instability of invariant sets and uniform boundedness and uniform ultimately boundedness of solutions for such systems. In the interests of brevity, not all of these results were proved. However, we provided references where some of the omitted proofs can be found. Next, we established converse theorems to some of the above results (specifically, necessary conditions for the uniform stability and uniform asymptotic stability of invariant sets), using some additional mild assumptions. These converse theorems show that under the given hypotheses, the sufficient conditions for uniform stability and uniform asymptotic stability of invariant sets established herein are as good as you can get.

The above results provide a basis for the qualitative analysis of important general classes of HDS's. To demonstrate this, we considered two such classes: sampled data control systems and systems with impulse effects.

\section{REFERENCES}

[1] P. J. Antsaklis, J. A. Stiver, and M. D. Lemmon, "Hybrid system modeling and autonomous control systems," in Hybrid Systems, R. Grossman, A. Nerode, A. Ravn, and H. Rischel, Eds. New York: Springer, 1993, pp. 366-392.

[2] A. Back, J. Guckenheimer, and M. Myers, "A dynamical simulation facility for hybrid systems," Hybrid Systems, R. Grossman, A. Nerode, A. Ravn, and H. Rischel, Eds. New York: Springer, 1993, pp. 255-267.

[3] R. W. Brockett, "Hybrid models for motion control systems," in Essays on Control Perspectives in the Theory and its Applications, H. L. Trentelman and J. C. Willems, Eds. Boston, MA: Birkhauser, 1993, pp. 29-53.

[4] A. Gollu and P. P. Varaiya, "Hybrid dynamical systems," in Proc 28th IEEE Conf. Decision and Control, Tampa, FL, Dec. 1989, pp. 2708-2712.

[5] R. Grossman, A. Nerode, A. Ravn, and H. Rischel, Eds., Hybrid Systems. New York: Springer, 1993.

[6] A. Nerode and W. Kohn, "Models for hybrid systems: Automata, topologies, controllability, observability," in Hybrid Systems, R. Grossman, A. Nerode, A. Ravn, and H. Rischel, Eds. New York: Springer, 1993, pp. 317-356.

[7] L. Tavernini, "Differential automata and their discrete simulators," Nonlinear Analysis, Theory, Methods, and Applications, vol. 11. New York: Pergamon, 1987, pp. 665-683.

[8] P. P. Varaiya, "Smart cars on smart roads: Problems of control," IEEE Trans. Automat. Contr., vol. 38, pp. 195-207, 1993.

[9] M. S. Branicky, V. S. Borkar, and S. K. Mitter, "A unified framework for hybrid control," in Proc. 33rd Conf. Decision and Control, Lake Buena Vista, FL, Dec. 1994, pp. 4228-4234.

[10] P. A. Iglesias, "On the stability of sampled-data linear time-varying feedback systems," in Proc. 33rd Conf. Decision and Control, Lake Buena Vista, FL, Dec. 1994, pp. 219-224.

[11] B. A. Francis and T. T. Georgiou, "Stability theory for linear timeinvariant plants with periodic digital controllers," IEEE Trans. Automat. Contr., vol. 33, pp. 820-832, 1988.

[12] T. Hagiwara and M. Araki, "Design of a stable feedback controller based on the multirate sampling of the plant output," IEEE Trans. Automat. Contr., vol. 33, pp. 812-819, 1988.

[13] K. M. Passino, A. N. Michel, and P. J. Antsaklis, "Lyapunov stability of a class of discrete event systems," IEEE Trans. Automat. Contr., vol. 39, pp. 269-279, Feb. 1994.

[14] M. Branicky, "Stability of switched and hybrid systems," in Proc. 33rd Conf. Decision and Control, Lake Buena Vista, FL, Dec. 1994, pp. 3498-3503.

[15] P. Peleties and R. DeCarlo, "Asymptotic stability of m-switched systems using Lyapunov-like functions," in Proc. American Control Conf., Boston, MA, June 1991, pp. 1679-1684. 
[16] A. N. Michel and K. Wang, Qualitative Theory of Dynamical Systems. New York: Marcel Dekker, 1995.

[17] T. Yoshizawa, Stability Theory by Lyapunov's Second Method. Tokyo, Japan: Math. Soc. Japan, 1966.

[18] V. I. Zubov, Methods of A. M. Lyapunov and Their Applications. Groningen, The Netherlands: P. Noordhoff Ltd., 1964.

[19] W. Hahn, Stability of Motion. Berlin, Germany: Springer-Verlag, 1967.

[20] R. K. Miller and A. N. Michel, Ordinary Differential Equations. New York: Academic, 1982.

[21] D. D. Bainov and P. S. Simeonov, Systems with Impulse Effect: Stability, Theory and Applications. New York: Halsted, 1989.

[22] H. Ye, A. N. Michel, and P. J. Antsaklis, "A general model for the qualitative analysis of hybrid dynamical systems," in Proc. 34th IEEE Conf. Decision and Control, New Orleans, LA, Dec. 1995, pp. 1473-1477.

[23] H. Ye, A. N. Michel, and L. Hou, "Stability theory for hybrid dynamical systems," in Proc. 34th IEEE Conf. Decision and Control, New Orleans, LA, Dec. 1995, pp. 2679-2684

[24] , "Stability analysis of discontinuous dynamical systems with applications," in Proc. 13th World Congr. Int. Federation of Automatic Control, vol. E-Nonlinear Systems, San Francisco, CA, June 1996, pp. $461-466$

[25] _ "Stability analysis of systems with impulse effects," in Proc. 35th IEEE Conf. Decision and Control, Kobe, Japan, Dec. 1996, pp. $159-161$.

[26] L. Hou, A. N. Michel, and H. Ye, "Some qualitative properties of sampled-data control systems," in Proc. 35th IEEE Conf. Decision and Control, Kobe, Japan, Dec. 1996, pp. 911-917.

[27] , "Stability analysis of switched systems," in Proc. 35th IEEE Conf. Decision and Control, Kobe, Japan, Dec. 1996, pp. 1208-1213.

[28] A. N. Michel and L. Hou, "Modeling and qualitative theory for general hybrid dynamical systems," in Proc. IFAC-IFIP-IMACS Conf. Control of Industrial Systems, Belfort, France, May 1997, vol. 3, pp. 173-183.

[29] M. S. Branicky, "Studies in hybrid systems: Modeling, analysis, and control," Ph.D. dissertation, MIT, Cambridge, MA, 1995.

[30] H. Ye, "Stability analysis of two classes of dynamical systems: General hybrid systems and neural networks with delays," Ph.D. dissertation, Univ. Notre Dame, May 1996

[31] T. Pavlidis, "Stability of systems described by differential equations containing impulses," IEEE Trans. Automat. Contr., vol. 12, pp. 43-45, Feb. 1967.

[32] A. T. Barabanov and Ye. F. Starozhilov, "Investigation of the stability of continuous discrete systems by Lyapunov's second method," Sov. J. Autom. Inf. Sci., vol. 21, no. 6, pp. 35-41, Nov./Dec. 1988.

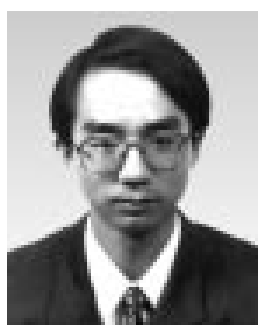

Hui Ye received the B.S. degree in mathematics from the University of Science and Technologies of China in 1990 and the M.S. degree in mathematics in 1992, the M.S. degree in electrical engineering in 1995, and the Ph.D. degree in electrical engineering in 1996, all from the University of Notre Dame, IN.

Currently he is with Lucent Technologies as a Member of Technical Staff to develop new technologies in wireless communications. His research interests include hybrid dynamic systems, artificial neural networks, and wireless communications.

Dr. Ye is a member of the Conference Editorial Board of the IEEE Control Society.

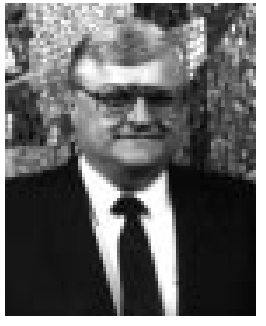

Anthony N. Michel (S'55-M'59-SM'79-F'82) received the Ph.D. degree in electrical engineering in 1968 from Marquette University, Milwaukee, WI, and the D.Sc. degree in applied mathematics from the Technical University of Graz, Austria, in 1973.

He has seven years of industrial experience. From 1968 to 1984 he was on the Electrical Engineering Faculty at Iowa State University, Ames. In 1984 he became Chair of the Department of Electrical Engineering, and in 1988 he became Dean of the College of Engineering at the University of Notre Dame, IN. He is currently the Frank M. Freimann Professor of Engineering and the Dean of the College of Engineering at Notre Dame. He has coauthored six books and several other publications.

Dr. Michel received (with R. D. Rasmussen) the 1978 Best Transactions Paper Award of the IEEE Control Systems Society, the 1984 Guillemin-Cauer Prize Paper Award of the IEEE Circuits and Systems Society (with R. K. Miller and B. H. Nam), and the 1993 Myril B. Reed Outstanding Paper Award of the IEEE Circuits and Systems Society. He was awarded the IEEE Centennial Medal in 1984, and in 1992 he was a Fulbright Scholar at the Technical University of Vienna. He received the 1995 Technical Achievement Award of the IEEE Circuits and Systems Society. He is a Past Editor of the IEEE TRAnsactions ON CiRcuits AND Systems (1981-1983) as well as a Past President (1989) of the Circuits and Systems Society. He is a Past Vice President of Technical Affairs (1994, 1995) and a Past Vice President of Conference Activities (1996, 1997) of the Control Systems Society. $\mathrm{He}$ is currently an Associate Editor at Large for the IEEE TRANSACTIONS ON Automatic Control, and he was Program Chair of the 1985 IEEE Conference on Decision and Control as well as General Chair of the 1997 IEEE Conference on Decision and Control. He was awarded an Alexander von Humboldt Forschungspreis (Research Award) for Senior U.S. Scientists (1998).

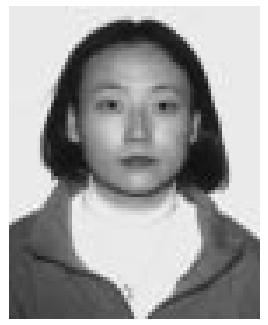

Ling Hou was born in Yanan, Shaanxi, China, in November 1972. She received the B.S. degree in mathematics from the University of Science and Technology of China in 1994 and the M.S. degree in electrical engineering from the University of Notre Dame, IN, in 1996, where she is currently a Ph.D. candidate in the Department of Electrical Engineering.

Her research interests include hybrid dynamical systems, discontinuous dynamical systems, systems with saturation, state estimation, and signal validation. 